\title{
Time-dependent operators on some non-orientable projective orbifolds*
}

\author{
R. S. Kraußhar ${ }^{\dagger}, \quad$ M.M. Rodrigues ${ }^{\ddagger}, \quad$ N. Vieira ${ }^{\ddagger}$ \\ ${ }^{\dagger}$ Fachgebiet Mathematik, \\ Erziehungswissenschaftliche Fakultät, Universität Erfurt \\ Nordhäuserstr. 63, 99089 Erfurt, Germany. \\ E-mail: soeren.krausshar@uni-erfurt.de \\ ¥ CIDMA - Center for Research and Development in Mathematics and Applications \\ Department of Mathematics, University of Aveiro \\ Campus Universitário de Santiago, 3810-193 Aveiro, Portugal. \\ E-mail: mrodrigues@ua.pt,nloureirovieira@gmail.com
}

\begin{abstract}
In this paper we present an explicit construction for the fundamental solution of the heat operator, the Schrödinger operator and related first order parabolic Dirac operators on a class of some conformally flat non-orientable orbifolds. More concretely, we treat a class of projective cylinders and tori where we can study parabolic monogenic sections with values in different pin bundles. We present integral representation formulas together with some elementary tools of harmonic analysis that enable us to solve boundary value problems on these orbifolds.
\end{abstract}

Keywords: Clifford and harmonic analysis; Heat operator; Schrödinger operator; Parabolic Dirac operator, Conformally flat orbifolds; Spin and pin structures; non-orientable manifolds.

MSC 2010: 30G35, 53C21, 53C27; 35J10; 35C15.

\section{Introduction}

The study of Dirac and Laplace operators on manifolds has lead to a profound understanding of many geometric aspects used in important physical models. The classical elliptic Dirac operator $D$ is a first order elliptic differential operator that acts on a vector bundle over a Riemannian spin manifold. It linearizes the second order Laplacian, viz $D^{2}=-\Delta$. An important advantage to use Dirac operators consists in the fact that they offer a well-developed function theory. Sections in the kernel of the Dirac operator satisfy a generalized Cauchy integral formula. This Cauchy integral formula in turn allows us to calculate the values of functions in terms of given boundary data arising from practical measurements. Based on these representations important existence and uniqueness theorems for the solutions of boundary value problems on manifolds could be established, see for instance $[4,8]$.Actually, to have an elliptic Dirac operator one not only needs a Riemannian structure but to also have a spin or pin structure (c.f. [19]).

In $[2,7,17]$ a parabolic version of the Dirac operator associated to a so-called Witt basis has been studied. This parabolic version of the Dirac operator now factorizes the heat operator and the Schrödinger operator. Many of the function theoretic tools can be carried over to this context and permitted us to tackle boundary value problems to the operator in the context of some Lorentzian manifolds.

Recently, one also started to carry over some of these techniques to the more general context of orbifolds. See for instance [10] and the references therein. Orbifolds naturally arise from factoring out simply connected

*The final version is published in Mathematical Methods in the Applied Science, Mathematical Methods in the Applied Sciences, 38-No.18, (2015), 5305-5319. It as available via the website http://onlinelibrary.wiley.com/doi/10.1002/mma.3492/abstract 
domains $G \subseteq \mathbb{R}^{n+1}$ by discrete Kleinian groups that act totally discontinuously on $G$. When the action is torsion free, which means that the group has no elements of finite order (except of the identity element), then we deal with a manifold. However, in general such a Kleinian group may admit torsion elements which causes singularities. The classical example is the quotient of complex upper half-plane by the modular group $S L(2, \mathbb{Z})$ and higher dimensional generalizations. An important property of the orbifolds (resp. manifolds) that arise by applying this construction is that they are conformally flat, that means they possess an atlas whose transition functions are conformal maps. In the more classical case dealing with the elliptic Dirac operator, the associated Cauchy kernel then can be expressed in terms of monogenic or harmonic automorphic forms on $\Gamma$ that are in the kernel of the elliptic Dirac or Laplace operator, respectively (c.f. $[14,16])$.

The goal of this paper is to continue the study of parabolic Dirac operators as well as related Heat and Schrödinger operators in the different context of some non-orientable conformally flat orbifolds.

It should be noticed that Dirac operators on non-orientable pin manifolds have been studied extensively in [3] and elsewhere. In this paper we now look at generalizations of cylinders and tori. Cylinders and tori, which arise by factoring out $\mathbb{R}^{n+1}$ by a discrete translation group, have natural non-orientable counterparts. Particularly, we look at a class of projective cylinders and tori, where we identify for example "upper" and "lower" parts with each other. This is translated into an additional symmetry structure which actually leads to a singularity - in general we are now dealing with orbifolds in this context. We explain how we can construct associated Cauchy kernels for the parabolic Dirac operator, as well as heat and Schrödinger kernels for this class of orbifolds. Similarly to the oriented case, we can consider distinct pin bundles in the non-oriented case. We show how arbitrary pin sections can be represented by these kernels. We also set up integral representation formulas for the sections in the kernel of the parabolic Dirac (resp. heat, Schrödinger) operator on these orbifolds.

This paper also provides a continuation of [15] where explicit formulas for the Cauchy and Green's kernels for the elliptic Dirac and Laplace operator have been developed.

The structure of the paper is as follows: in the section "Preliminaries" we recall some basic facts about Clifford algebras and about factorization and regularization of time-dependent operators. In Section 3, we define the spin and the pin structures that are necessary for the development of our work. In the following section we study the non-orientable projective counterparts of cylinders and tori. We start by presenting the geometric context. Then we construct the Cauchy and heat kernels, as well as the corresponding integral formulas. Section 5 is dedicated to the study of the Schrödinger operator. Here we start by studying the inhomogeneous Schrödinger equation in orbifolds in terms of a proper Teodorescu and Cauchy operators. In this last section we finally present a Hodge-type decomposition for the regularized case and we will study the behavior of our result when the regularization parameter tends to zero.

\section{Preliminaries}

\subsection{Clifford algebras}

As usual we denote the orthonormal basis of $\mathbb{R}^{n}$ by $\left\{e_{1}, \cdots, e_{n}\right\}$. We define the universal real Clifford algebra $C \ell_{0, n}$ as the $2^{n}$-dimensional associative algebra which obeys the multiplication rules $e_{i} e_{j}+e_{j} e_{i}=-2 \delta_{i, j}$. A vector space basis for $C \ell_{0, n}$ is generated by the elements $e_{0}=1$ and $e_{A}=e_{h_{1}} \cdots e_{h_{k}}$, where $A=\left\{h_{1}, \ldots, h_{k}\right\} \subset$ $M=\{1, \ldots, n\}$, for $1 \leq h_{1}<\cdots<h_{k} \leq n$. Each $x \in C \ell_{0, n}$ can be represented by $x=\sum_{A} x_{A} e_{A}, x_{A} \in \mathbb{R}$. The Clifford conjugation is defined by $\overline{1}=1, \overline{e_{j}}=-e_{j}$ for all $j=1, \ldots, n$, and we have $\overline{a b}=\bar{b} \bar{a}$. We introduce the complexified Clifford algebra $\mathbb{C}_{n}$ as the tensor product

$$
\mathbb{C} \otimes C \ell_{0, n}=\left\{w=\sum_{A} w_{A} e_{A}, w_{A} \in \mathbb{C}, A \subset M\right\},
$$

where the imaginary unit $i$ of $\mathbb{C}$ commutes with the basis elements, i.e., $i e_{j}=e_{j} i$ for all $j=1, \ldots, n$. To avoid ambiguities with the Clifford conjugation, we denote the complex conjugation mapping a complex scalar $w_{A}=a_{A}+i b_{A}$, with real components $a_{A}$ and $b_{A}$, onto $\bar{w}_{A}=a_{A}-i b_{A}$ by $\sharp$. Note, $e_{j}^{\sharp}=e_{j}$ for all $j=1, \ldots, n$. We have a pseudonorm on $\mathbb{C}_{n}$ viz $|w|:=\sum_{A}\left|w_{A}\right|$ where $w=\sum_{A} w_{A} e_{A}$. Notice that for $a, b \in \mathbb{C}_{n}$ we only have $|a b| \leq 2^{n}|a||b|$.

A function $u: U \mapsto \mathbb{C}_{n}$ has a representation $u=\sum_{A} u_{A} e_{A}$ with $\mathbb{C}$-valued components $u_{A}$. Properties such as continuity will be understood componentwise. The Euclidean Dirac operator $D=\sum_{j=1}^{n} e_{j} \partial_{x_{j}}$ factorizes 
the Euclidean Laplacian viz $D^{2}=-\Delta=-\sum_{j=1}^{n} \partial x_{j}^{2}$. Next we recall that a $\mathbb{C}_{n}$-valued and differentiable function defined on an open set $U \subseteq \mathbb{R}^{n}, u: U \mapsto \mathbb{C}_{n}$, is called left-monogenic if it satisfies $D u=0$ on $U$ (resp. right-monogenic if it satisfies $u D=0$ on $U$ ).

Following along the line of investigation presented in [7], to address time dependent problems we embed $\mathbb{R}^{n}$ into $\mathbb{R}^{n+2}$ by adding to further basis elements $\mathfrak{f}$ and $\mathfrak{f}^{\dagger}$ that satisfy

$$
\mathfrak{f}^{2}=\mathfrak{f}^{\dagger^{2}}=0, \quad \quad \mathfrak{f f}^{\dagger}+\mathfrak{f}^{\dagger} \mathfrak{f}=1, \quad \mathfrak{f} e_{j}+e_{j} \mathfrak{f}=\mathfrak{f}^{\dagger} e_{j}+e_{j} \mathfrak{f}^{\dagger}=0, \quad j=1, \cdots, n .
$$

The extended basis is called Witt basis. This construction allows us to apply a suitable factorization of time evolution operators where only partial derivatives are used (for more details see $[6,7,17]$ ).

We consider $\mathbb{C}_{n}$-valued maps from a bounded time-dependent domain $\Omega=\underline{\Omega} \times I \subseteq \mathbb{R}^{n} \times \mathbb{R}^{+}$with a piecewise smooth boundary $\Gamma=\partial \Omega$, i.e., functions in the variables $\left(x_{1}, x_{2}, \ldots, x_{n}, t\right)$ where $x_{i} \in \mathbb{R}$ for $i=1, \ldots, n$, $t \in I=\left[0, T\left[\right.\right.$. For the sake of readability, we abbreviate the space-time tuple $\left(x_{1}, x_{2}, \ldots, x_{n}, t\right)$ simply by $(x, t)$, where we identify $x=x_{1} e_{1}+\cdots+x_{n} e_{n}$. For additional details on Clifford analysis, we refer to [12, 13] and elsewhere.

\subsection{Time-dependent operators: factorization and regularization procedure}

Let us consider the notation $\Omega_{t}=\{x:(x, t) \in \underline{\Omega} \times I\} \subset \mathbb{R}^{n}$. Following the ideas of $[2,7,17]$ we introduce the definition:

Definition 2.1 For a function $u \in \stackrel{\circ}{W_{p}^{1}}(\Omega)$ we define the forward/backward parabolic Dirac operator as

$$
D_{ \pm} u=\left(D+\mathfrak{f} \partial_{t} \pm \alpha \mathfrak{f}^{\dagger}\right) u .
$$

Applying the multiplication rules (1) we get the factorization of a family of time-dependent operators, i.e.,

$$
\left(D_{ \pm}\right)^{2} u=\left(-\Delta \pm \alpha \partial_{t}\right) u
$$

From $[2,7,17]$ we have that the fundamental solution of $D_{+}$reads

$$
E_{+}(x, t)=e_{+}(x, t) D_{+}=\frac{H(t)}{(4 \pi \alpha t)^{\frac{n}{2}}} \exp \left(-\alpha \frac{|x|^{2}}{4 t}\right)\left(-\frac{x}{2 t}+\mathfrak{f}\left(\frac{|x|^{2}}{4 t^{2}}+\frac{n}{2 t}\right)+\alpha \mathfrak{f}^{\dagger}\right),
$$

where $H(t)$ denotes the Heaviside function and

$$
e_{+}(x, t)=\alpha e(x, t)=\frac{\alpha H(t)}{(4 \pi \alpha t)^{\frac{n}{2}}} \exp \left(-\alpha \frac{|x|^{2}}{4 t}\right)
$$

is the fundamental solution of $-\Delta+\alpha \partial_{t}$. The kernels (3) and (4) can be used to solve special boundary value problems. See for instance [13] for more details.

In the Schrödinger case $(\alpha=i)$, the operator $-\Delta+i \partial_{t}$ has the following fundamental solution

$$
e_{+}(x, t)=i e(x, t)=\frac{i H(t)}{(4 \pi i t)^{\frac{n}{2}}} \exp \left(-i \frac{|x|^{2}}{4 t}\right) .
$$

Obviously, this function has singularities at all the points of the hyperplane $t=0$. This is a substantial difference to the context of hypoellitic operators [1] where one deals with an isolated point singularity only. Moreover, these singularities are not removable by standard methods. This property causes additional problems in the study of some integral operators or series that are constructed on the basis of these functions; we cannot guarantee the convergence in the classical sense. In order to overcome this problem we need to regularize the fundamental solution and the associated integral operators (see [5, 17, 22]). In the representation of the operators and their associated fundamental solutions, one has to substitute the imaginary unit by the parameter $\mathbf{k}=\frac{\epsilon+i}{\epsilon^{2}+1}$. Hence, we obtain a family of regularized operators and associated regularized fundamental solutions which are locally integrable in $\mathbb{R}^{n} \times \mathbb{R}_{0}^{+} \backslash\{(0,0)\}$. Following [1], for each $\epsilon>0$, the operator $\left(-\Delta+\mathbf{k} \partial_{t}\right)$ is a hypoelliptic operator. It ensures the good convergence behavior for the integral operators as well as for the associated function series representations, as we shall see later on. The fundamental solution for this operator is given by

$$
e_{+}^{\epsilon}(x, t)=(\epsilon+i) e(x,(\epsilon+i) t)=(\epsilon+i) \frac{H(t)}{(4 \pi(\epsilon+i) t)^{\frac{n}{2}}} \exp \left(-\frac{(\epsilon-i)|x|^{2}}{4\left(\epsilon^{2}+1\right) t}\right), \quad \epsilon>0 .
$$

This motivates us to consider the following regularized parabolic-type Dirac operator [5]. 
Definition 2.2 For a function $u \in W_{p}^{a}(\Omega)$ with $1<p<\infty$ and $a \in \mathbb{N}$, we define the forward/backward regularized parabolic-type Dirac operator by

$$
D_{ \pm}^{\epsilon} u=\left(D+\mathfrak{f} \partial_{t} \pm \mathbf{k} \mathfrak{f}^{\dagger}\right) u,
$$

where D stands for the usual (spatial) Euclidean Dirac operator.

This operator factorizes the associated backward regularized Schrödinger operator, i.e.,

$$
\left(D_{ \pm}^{\epsilon}\right)^{2} u=\left(-\Delta \pm \mathbf{k} \partial_{t}\right) u .
$$

This regularized operator has the mapping property $D_{ \pm}^{\epsilon}: W_{p}^{1}(\Omega) \rightarrow L_{p}(\Omega)$. For more details about this regularization procedure, we refer the reader to $[5,23]$. In [5] the authors proved the following result:

Theorem 2.3 For the sequence of parabolic-type Dirac operators $D_{-}^{\epsilon}$, with $\epsilon>0$, we have the following convergence

$$
\left\|D_{ \pm}-D_{ \pm}^{\epsilon}\right\|_{L_{1}(\Omega)} \rightarrow 0 .
$$

We now present the family of regularized fundamental solutions for this first-order operator.

Definition 2.4 Let $e_{+}^{\epsilon}=e_{+}^{\epsilon}(x, t)$ be a fundamental solution of the operator (6). Then the function $E_{+}^{\epsilon}(x, t)=$ $e_{+}^{\epsilon}(x, t) D_{+}^{\epsilon}$ is a fundamental solution for the operator $D_{+}^{\epsilon}$

Simple calculations (see [5]) give

$$
E_{+}^{\epsilon}(x, t)=e_{+}^{\epsilon}(x, t) D_{+}^{\epsilon}=e_{+}^{\epsilon}(x, t)\left[\frac{-x}{2(\epsilon+i) t}+\mathfrak{f}\left(\frac{-n}{2 t}+\frac{|x|^{2}}{4(\epsilon+i) t^{2}}\right)+\mathbf{k} \mathfrak{f}^{\dagger}\right] .
$$

In [5] the authors proved the following regularized Borel-Pompeiu type formula

$\int_{\partial \Omega} E_{+}^{\epsilon}\left(x-x_{0}, t-t_{0}\right) d \sigma_{x, t} u(x, t)=u\left(x_{0}, t_{0}\right)+\int_{\Omega} E_{+}^{\epsilon}\left(x-x_{0}, t-t_{0}\right)\left(D_{-}^{\epsilon} u\right)(x, t) d x d t, \quad\left(x_{0}, t_{0}\right) \notin \partial \Omega$.

Here, the surface element is given by the contraction of the homogeneous operator associated to $D_{-}^{\epsilon}$ with the volume element, i.e., $\left.d \sigma_{x, t}=\left(D_{x}+\mathfrak{f} \partial_{t}\right)\right\rfloor d x d t$ (for more details about this definition see [7, 9]). Moreover, for $u \in \operatorname{Ker}\left(D_{-}^{\epsilon}\right)$ the authors presented the following regularized Cauchy type integral formula

$$
\int_{\partial \Omega} E_{+}^{\epsilon}\left(x-x_{0}, t-t_{0}\right) d \sigma_{x, t} u(x, t)=u\left(x_{0}, t_{0}\right) .
$$

For more details on the application of this regularization procedure to the Schrödinger operator in the context of classical Clifford analysis, see for instance [5] and [23].

\section{Spin and Pin structures}

Following the ideas of our previous papers $[16,17]$, we briefly recall that conformally flat manifolds are in general $n$-dimensional manifolds that possess atlases whose transition functions are conformal maps. For $n \geq 3$ the set of conformal maps coincides with the set of Möbius transformations representing reflections at spheres and hyperplanes. From the constructive group theoretic point of view it makes sense to consider in this context more generally conformally flat orbifolds instead of manifolds only. This framework admits some singularities in the geometries.

As mentioned for instance in the classical work by N. Kuiper [18] concrete examples of conformally flat orbifolds can be constructed by factoring out a simple connected domain $X$ by a Kleinian group $\Gamma$ that acts discontinuously on $X$. The classical example of factoring out upper half-plane by the modular group $S L(2, \mathbb{Z})$ fits within this context as a special case.

In the particular cases where $\Gamma$ is torsion free, the topological quotient $X / \Gamma$, consisting of the orbits of a pre-defined group action $\Gamma \times X \rightarrow X$, is endowed with a differentiable structure. Then we deal with examples of conformally flat manifolds. 
A classical way of obtaining pin or spin structures for a given Riemannian manifold is to look for a lifting of the principle bundle associated to the orthogonal group $O(n)$ or the special orthogonal group $S O(n)$, to a principle bundle for the pin group $\operatorname{Pin}(n)$ or spin group $\operatorname{Spin}(n)$. The $\operatorname{Spin}(n)$ group is a subgroup of $\operatorname{Pin}(n)$ of index 2. Furthermore, $\operatorname{Spin}(n)$ is a double cover of $S O(n)$. So there is a surjective homomorphism $\theta: \operatorname{Spin}(n) \rightarrow S O(n)$ with kernel $\mathbb{Z}_{2}=\{ \pm 1\}$ (for basic facts on spin and pin structures we refer [9]). As explained in Appendix $\mathrm{C}$ of [20] this gives rise to a choice of two local liftings of the principle $S O(n)$ bundle to a principle $\operatorname{Spin}(n)$ bundle. The number of different global liftings is given by the number of elements in the cohomology group $H^{1}\left(M, \mathbb{Z}_{2}\right)$. These choices of liftings give rise to different spinor bundles over $M$. Similarly, $\operatorname{Pin}(n)$ is a double cover of $O(n)$ and analogously different pin bundles are obtained. Following [21] we may now talk about monogenic (harmonic) sections.

Definition 3.1 Let $M$ be a conformally flat spin manifold with spinor bundle $E$. Then a section $f: M \rightarrow E$ is called a left monogenic (harmonic) section if locally $f$ reduces to a left monogenic (harmonic) function.

The associated Laplacian on such a spin manifold is also called the spinorial Laplacian. Not all conformally flat manifolds are spin manifolds. A typical counterexample is the real projective space $\mathbb{R} P^{n}=S^{n} /\{ \pm 1\}$.

When $n$ is even, $\mathbb{R} P^{n}$ is no longer orientable. However, $\mathbb{R} P^{n}$ does still admit pin structures in these cases and hence pin bundles similar to those described earlier in this section. Classical examples of conformally flat spin manifolds are the cylinders and tori considered in $[2,16,17]$. Here we considered the $n$-dimensional orthonormal lattice

$$
\mathbb{Z}_{n}:=\mathbb{Z} e_{1}+\cdots+\mathbb{Z} e_{n}
$$

and the group action

$$
\mathbb{Z}_{n} \times \mathbb{R}^{n} \rightarrow \mathbb{R}^{n}, v \circ x \mapsto x+v, \quad v \in \mathbb{Z}_{n}, x \in \mathbb{R}^{n} .
$$

The invariance group of this lattice is the discrete translation group generated by the special Möbius transformations $\psi(x)=x+e_{i}$ for $i=1, \ldots, n$.

Since $\mathbb{Z}_{n}$ is a torsion free discrete Kleinian group, the topological quotient $T_{n}:=\mathbb{R}^{n} / \mathbb{Z}_{n}$ consisting of the orbits of the above defined group action actually is a conformally flat manifold in $n$ real variables. It represents an $n$-dimensional torus. Of course any subgroup of a torsion free Kleinian group is again torsion free. Let $\Omega_{k}$ be a $k$-dimensional sublattice of $\mathbb{Z}_{n}$. So, in particular for all $k=1, \ldots, n-1$ the quotient sets $C_{k}:=\mathbb{R}^{n} / \mathbb{Z}_{k}$ which arise by the same group action are conformally flat manifolds, too. While $C_{n}=: T_{n}$ (the $n$-torus) is compact, the other manifolds $C_{k}$ with $k=1, \ldots, n-1$ are non-compact and have $n-k$ unbounded directions. We call them $k$-cylinders. The classical infinite cylinder is obtained by $\mathbb{R}^{2} / \mathbb{Z} e_{1}$. It arises from the group action $\mathbb{Z} \times \mathbb{R}^{2} \rightarrow \mathbb{R}^{2}$ defined by $m \circ(x, y) \mapsto(x+m, y)$, where $m \in \mathbb{Z}$ and $(x, y) \in \mathbb{R}^{2}$.

Next, following [16], the decomposition of the lattice $\mathbb{Z}_{k}$ into the direct sum of the sublattices $\mathbb{Z}_{l}:=\mathbb{Z} v_{1}+$ $\cdots+\mathbb{Z} v_{l}$ and $\mathbb{Z}_{n-l}:=\mathbb{Z} v_{l+1}+\cdots+\mathbb{Z} v_{k}$ gives rise to $2^{k}$ distinct spinor bundles on $C_{k}$ (that we will denote by $\left.E^{(l)}\right)$ by making the identification $(x, X) \leftrightarrow\left(x+\underline{m}+\underline{n},(-1)^{m_{1}+\cdots+m_{l}} X\right)$. Here $x \in \mathbb{R}^{n}$ and $X \in C l_{n}$. Here, we use the notation $\underline{m}=m_{1} v_{1}+\cdots+m_{l} v_{l} \in \mathbb{Z}_{l}$ and $\underline{n}:=n_{l+1} v_{l+1}+\cdots+n_{k} v_{k} \in \mathbb{Z}_{k-l}$ where $m_{1}, \ldots, m_{k} \in \mathbb{Z}$. In all that follows we denote the trivial bundle by $E^{(1)}$. Since the manifolds $C_{k}$ are orientable, we are dealing with examples of spin manifolds in this context here.

Notice that the different spin structures on a spin manifold $M$ are detected by the number of distinct homomorphisms from the fundamental group $\Pi_{1}(M)$ to the group $\mathbb{Z}_{2}$. In the case of the $k$-cylinder $C_{k}$ we have that $\Pi_{1}\left(C_{k}\right)=\mathbb{Z}^{k}$. There are two homomorphisms of $\mathbb{Z}$ to $\mathbb{Z}_{2}$. The first is $\theta_{1}: \mathbb{Z} \rightarrow \mathbb{Z}_{2}: \theta_{1}(k) \equiv 0 \bmod 2$ while the second is the homomorphism $\theta_{2}: \mathbb{Z} \rightarrow \mathbb{Z}_{2}: \theta_{2}(k) \equiv 1 \bmod 2$. Consequently, there are $2^{k}$ distinct spin structures on $C_{k}$. Consequently the $n$-torus $C_{n}=T_{n}$ has $2^{n}$ distinct spin structures. $T_{n}$ is also an example of a Bieberbach manifold. Further details of spin structures on the $n$-torus and other Bieberbach manifolds can be found in [11].

In the following section we explain how we can adapt the construction method that we presented in this subsection in order to deal with some non-orientable counterparts of the manifolds considered here which can be constructed from the same Kleinian groups by which we constructed these cylinders and tori. 


\section{Non-orientable projective counterparts of cylinders and tori}

\subsection{The geometric context}

The oriented cylinder $C$ defined as the topological quotient $\mathbb{R}^{2} / \mathbb{Z}$ has several natural non-oriented projective counterparts. There classical projective cylinder can be constructed from the ordinary infinite cylinder by identifying the "northern" hemisphere with the "southern" hemisphere. In this case one has an additional symmetry relation on the original topological cylinder. This relation is mathematically expressed by $f(x, y)=$ $f(x,-y)$ in addition to the usual periodic relation $f(x+m, y)=f(x, y)(m \in \mathbb{Z})$. Recall that the standard cylinder is constructed by gluing the two vertices of a two-dimensional strip of length 1 in a straight parallel way together without performing any twists. In the case of the classical projective cylinder, one has besides the usual group action on the $x$-component of the form $\mathbb{Z} \times \mathbb{R}^{2} \rightarrow \mathbb{R}^{2}, m \circ(x, y)=(x+m, y)$ an additional group action $\mathbb{Z}_{2} \times \mathbb{R} \rightarrow \mathbb{R}$ on the second component, mapping $y$ and $-y$ on the same value.

Functions on the projective standard cylinders consequently have an additional invariance under the action of the group $\{ \pm 1\} \cong \mathbb{Z}_{2}$ in the $y$-component. Notice that already this classical example is not a classical manifold anymore. It is the half of the infinite cylinder and hence has the structure of a manifold with boundary. It is not orientable either.

In the $n$-dimensional setting we can construct in a similar way a number of non-oriented analogues of the oriented $k$-cylinders defined by $C_{k}:=\mathbb{R}^{n} / \Omega_{k}$ where $k \in\{1, \ldots, n-1\}$. Here and in all that follows, $\Omega_{k} \subset \mathbb{R}^{k}$ denotes a $k$-dimensional lattice spanned entirely by $k \mathbb{R}$-linearly independent reduced vectors $\underline{\omega}_{1}, \ldots, \underline{\omega}_{k} \in \mathbb{R}^{k}$.

Now let $\underline{x}$ be a reduced vector from $\mathbb{R}^{k}$. Suppose that $\underline{\omega}:=m_{1} \underline{\omega}_{1}+\cdots+m_{k} \underline{\omega}_{k}$ is a vector from the lattice $\Omega_{k}$. Further let $p$ be an integer from the set $\{k+1, \ldots, n\}$ in all that follows. We write a vector $x=x_{1} e_{1}+\cdots x_{k} e_{k}+x_{k+1} e_{k+1}+\cdots+x_{p} e_{p}+x_{p+1} e_{p+1}+\cdots x_{n} e_{n}$, where $1<k<p<n$. The first part $x_{1} e_{1}+\cdots x_{k} e_{k}$ will be abbreviated by $\underline{x}$. The other components are denoted by $x_{k+1}, \ldots, x_{p}, \ldots, x_{n}$. Next let $X \in C l_{n}$. Now, by identifying the tupel

$$
\left(\underline{x}+\underline{\omega}, x_{k+1}, . ., x_{p}, . ., x_{n}, X\right)
$$

with

$$
\left(\underline{x},-x_{k+1}, \ldots,-x_{p}, \ldots, x_{p+1}, \ldots, x_{n}, X\right)
$$

we obtain a class of conformally flat orbifolds denoted by $\mathcal{M}_{k, p}$. Here $\Gamma=\mathbb{Z}^{k} \times \mathbb{Z}_{2}$. Notice that we are not dealing with manifolds here, either. The space $\mathbb{R}^{n}$ splits as the product

$$
\mathbb{R}^{n} \cong \mathbb{R}^{k} \times \mathbb{R}^{p-k} \times \mathbb{R}^{n-p} .
$$

The left part of $\Gamma$ acts on $\mathbb{R}^{k}$; the right part acts on $\mathbb{R}^{p-k}$. The both parts act independently of each other, hence the quotient is the product $\left(\mathbb{R}^{k} / \mathbb{Z}^{k}\right) \times\left(\mathbb{R}^{p-k} / \mathbb{Z}_{2}\right) \times \mathbb{R}^{n-p}$. The first term in the product is a torus. But the second part has a singularity at the origin. It is an orbifold and not a manifold. Notice further that these orbifolds are non-orientable either and hence not spin. In the case of not having any sign changes at all, we again deal with the class of oriented conformally flat cylinders and tori which we briefly discussed in our preceding papers.

We can say more. Analogously to the case of a spin manifold we can set up several distinct pin bundles. One pin bundle which is different from the trivial one given above is obtained by identifying the pair

$$
\left(\underline{x}+\underline{\omega}, x_{k+1}, \ldots, x_{n}, X\right)
$$

with

$$
\left(\underline{x},-x_{k+1}, \ldots,-x_{p}, x_{p+1}, \ldots, x_{n},-X\right) .
$$

Other distinct choices arise by again splitting the period lattice $\Omega_{k}$ into two sublattices $\Omega_{k}=\Omega_{l} \oplus \Omega_{k-l}$ where $1<l<k$. Writing an element $\underline{\omega} \in \Omega_{k}$ in the form $\underline{\omega}=\underline{m}+\underline{n}$ with $\underline{m}=m_{1} \underline{\omega}_{1}+\cdots+m_{l} \underline{\omega}_{l} \in \Omega_{k}$ and $\underline{n}=m_{l+1} \underline{\omega}_{l+1}+\cdots+m_{k} \underline{\omega}_{k} \in \Omega_{k-l}$ gives rise to again consider the following identifications

$$
\left(\underline{x}+\underline{\omega}, x_{k+1}, \ldots, x_{n}, X\right)
$$

with

$$
\left(\underline{x},-x_{k+1}, \ldots,-x_{p}, x_{p+1} \ldots, x_{n},(-1)^{m_{1}+\cdots+m_{l}} X\right) .
$$




\subsection{Construction of the Cauchy and heat kernels and associated integral formulas}

For simplicity let us look in the following at the trivial pin bundle of the orbifold $\mathcal{M}_{k, p}$ in $\mathbb{R}^{n} \times \mathbb{R}^{+}$, where we simply identify

$$
\left(\underline{x}+\underline{\omega}, x_{k+1}, \ldots, x_{p}, x_{p+1}, \ldots, x_{n}, X, t\right)
$$

with

$$
\left(\underline{x},-x_{k+1}, \ldots,-x_{p}, x_{p+1}, \ldots, x_{n}, X, t\right) .
$$

Adapting the arguments from [2] one can express the parabolic Dirac operator on the oriented $k$-cylinder by the infinite $k$-fold periodization of the kernel function $E_{+}(x, t)$ over the period lattice, i.e.

$$
g_{k}(x, y, t)=\sum_{\underline{\omega} \in \Omega_{K}} E_{+}(x-y+\underline{\omega}, t) .
$$

To explain the convergence of the series let us consider for simplicity the case of the orthonormal lattice $\Omega_{k}=\mathbb{Z} e_{1}+\cdots+\mathbb{Z} e_{k}$. The general case can easily be adapted by either applying properly chosen rotations and dilations or by adapting the below explained decomposition of the lattice accordingly. In the case of the orthonormal lattice, the latter is decomposed in the form

$$
\Omega_{k, m}:=\left\{\omega \in \Omega_{k} \mid\|\omega\|_{\max }=m\right\}
$$

Define the subsets

$$
L_{k}:=\left\{\omega \in \Omega_{k} \mid\|\omega\|_{\max }<m\right\},
$$

which are such that $\sharp L_{k, m}=(2 m+1)^{k}$ and $\sharp \Omega_{k, m}=(2 m+1)^{k}-(2 m-1)^{k}$. To show the normal convergence one takes an arbitrary compact subset $K \subset \mathbb{R}^{n}$. Then there is an $r>0$ such that all $x \in K$ satisfy $\|x\|_{\text {max }}<$ $\|x\|_{2}<r$. Without loss of generality consider the summation over the lattice points satisfying $\|\omega\|_{\max }>[r]+1$. Note that $\|x+\omega\|_{2}>\|\omega\|_{2}-\|x\|_{2}>\|\omega\|_{\max }-\|x\|_{2}=m-\|x\|_{2}>m-r$. Therefore, with a properly chosen positive constant $C$ and proper polynomials $P$ and $P^{\prime}$ we obtain that

$$
\begin{aligned}
& \sum_{m=[r]+1}^{\infty} \sum_{\omega \in \Omega_{k}, m}\left\|E_{+}(x+\omega, t)\right\|_{2} \\
& \quad<\frac{C}{(2 \sqrt{\pi t})} \sum_{m=[r]+1}^{\infty} \sum_{\omega \in \Omega_{k, m}}\left\|-\frac{x+\omega}{2 t}+\mathfrak{f}\left(\frac{|x+\omega|^{2}}{4 t}+\frac{n}{2 t}\right)+\mathfrak{f}^{\dagger}\right\|_{2} e^{-\frac{\|x+\omega\|_{2}^{2}}{4 t}} \\
& \quad<\frac{C}{(2 \sqrt{\pi t})} \sum_{m=[r]+1}^{\infty}\left[(2 m+1)^{k}-(2 m-1)^{k}\right]\left(\frac{r+m \sqrt{n}}{2 t}+\frac{(r+m \sqrt{n})^{2}}{4 t}+\frac{n}{2 t}+1\right) e^{-\frac{(m-r)^{2}}{4 t}} .
\end{aligned}
$$

where we applied that $\|x+\omega\|_{2}<\|x\|_{2}+\|\omega\|_{2}<r+m \sqrt{n}$. It is clear that the latter expression converges since the exponential decreasing term dominates the growth of any polynomial. So, applying Weierstraß' convergence theorem, the expression $g_{k}(x, y, t)$ is a well-defined null-solution to the parabolic Dirac equation. Per construction, it is $k$-fold periodic with respect to the period lattice $\Omega_{k}$.

More precisely, the application of the canonical projection map $p: \mathbb{R}^{n+1} \rightarrow C_{k}, x \mapsto x \bmod \Omega_{k}$ to this periodized kernel, i.e. $G_{k}\left(x^{\prime}, y^{\prime}, t\right):=p\left(g_{k}(x, y, t)\right)$ then gives rise to the Cauchy kernel of $D_{-}$on the $k$-cylinder $C_{k}$. Analogously, the heat kernel on $C_{k}$ can be obtained by applying that projection map to the series

$$
h_{k}(x, y, t)=\sum_{\underline{\omega} \in \Omega_{K}} e_{+}(x-y+\underline{\omega}, t),
$$

i.e. $H_{k}\left(x^{\prime}, y^{\prime}, t\right):=p h_{k}(x, y, t)$. Here, the proof is even simpler, because in this context both polynomials $P$ and $P^{\prime}$ can be chosen identically equal to 1 . To address the projective versions of th cylinders, we now can express the Cauchy kernel of the parabolic Dirac operator and the heat kernel of the heat operator on the orbifold $\mathcal{M}_{k, p}$ by a finite superposition of the fundamental sections $G_{k}$ or $H_{k}$, respectively, taking care of the additional minus signs in the coordinate directions $k+1, \ldots, p$. More concretely, we may establish that 
Theorem 4.1 The Cauchy kernel of the parabolic Dirac operator on $\mathcal{M}_{k, p}$ associated to the trivial pin bundle can be expressed in the form

$G_{\mathcal{M}_{k, p}}\left(x^{\prime \prime}, y^{\prime \prime}, t\right)=P_{k, p}\left(\sum_{\varepsilon_{k+1}, \ldots, \varepsilon_{p} \in\{ \pm 1\}} g_{k}\left(\underline{x^{\prime}}-\underline{y}^{\prime}, \varepsilon_{k+1}\left(x_{k+1}^{\prime}-y_{k+1}^{\prime}\right), \varepsilon_{p}\left(x_{p}^{\prime}-y_{p}^{\prime}\right),\left(x_{p+1}^{\prime}-y_{p+1}^{\prime}\right), \ldots\left(x_{n}^{\prime}-y_{n}^{\prime}\right), t\right)\right)$.

Here, $P_{p}$ denotes the projection from the fully infinite cylinder $C_{k}$ down to the projective cylinder $\mathcal{M}_{k, p}$. In the cases of the other pin bundles, we need to add the corresponding minus sign in the sum in front of the multiperiodic expression $G_{k}$. In the case of the orthonormal lattice the kernel then reads

$$
\begin{aligned}
G_{\mathcal{M}_{k, p}}\left(x^{\prime \prime}, y^{\prime \prime}, t\right)=P_{k, p} & \left(\sum_{\varepsilon_{k+1}, \ldots, \varepsilon_{p} \in\{ \pm 1\}} \sum_{\omega=m_{1} e_{1}+\cdots+m_{k} e_{k} \in \mathbb{Z}^{l} \times \mathbb{Z}^{k-l}}(-1)^{m_{1}+\cdots m_{l}}\right. \\
& \left.\times E_{+}\left(\underline{x^{\prime}}-\underline{y^{\prime}}-\underline{\omega}, \varepsilon_{k+1}\left(x_{k+1}^{\prime}-y_{k+1}^{\prime}\right), \ldots, \varepsilon_{p}\left(x_{p}^{\prime}-y_{p}^{\prime}\right),\left(x_{p+1}^{\prime}-y_{p+1}^{\prime}\right), \ldots\left(x_{n}^{\prime}-y_{n}^{\prime}\right), t\right)\right) .
\end{aligned}
$$

To prove this statement we first recall that in the preceding works [2] it has already been shown that the subseries $G_{k}(x, y, t)$ are well-defined and normally convergent in $\mathbb{R}^{n} \backslash \Omega_{k}$. Furthermore, they are invariant under the lattice transformations $G_{k}\left(x+v_{i}, y, t\right)=G_{k}\left(x, y+v_{i}, t\right)=G_{k}(x, y, t)$ for all $v_{i} \in \Omega_{k}$, and therefore for all $v \in \Omega_{k}$. The summation over all $\varepsilon_{k+1}, \ldots, \varepsilon_{p} \in\{ \pm 1\}$ is a finite sum of expressions $G_{k}(x, y, t)$. Consequently, the total series again converges normally in $\mathbb{R}^{n} \backslash \Omega_{k}$ and is $\Omega_{k}$-invariant. Due to the extension of the summation over all possible sign combinations in the coordinates $k+1, \ldots, p$ the outcome then is also invariant under transformations of the form

$$
\begin{aligned}
f\left(\underline{x}-\underline{y},\left(x_{k+1}-y_{k+1}\right), \ldots,\left(x_{p}-y_{p}\right), \ldots,\left(x_{n}-y_{n}\right), t\right) \\
\quad \mapsto f\left(\underline{x}-\underline{y},-\left(x_{k+1}-y_{k+1}\right), \ldots,-\left(x_{p}-y_{p}\right),\left(x_{p+1}-y_{p+1}\right), \ldots,\left(x_{n}-y_{n}\right), t\right) .
\end{aligned}
$$

Therefore, the projection $P_{k, p}:=P_{p} \circ p_{k}$ of the entire series descends to a well-defined left parabolic monogenic section on $\mathcal{M}_{k, p}$. On the orbifold $\mathcal{M}_{k, p}$ the kernel then has exactly one point singularity of the order of the usual Cauchy kernel. As a sum of left monogenic functions, it is again left monogenic except at this point singularity. As a consequence of the usual Cauchy theorem valid in the universal covering space $\mathbb{R}^{n}$, cf. [12, 13], which states that the oriented boundary integral over a left monogenic function vanishes identically, we can deduce a properly adapted version of Cauchy's integral formula on the orbifold from which then follows that $G_{\mathcal{M}_{k, p}}\left(x^{\prime \prime}, y^{\prime \prime}, t\right)$ actually is the Cauchy kernel.

To get there, let us first consider a strongly Lipschitz hypersurface $S$ that lies completely in that part of the standard fundamental period parallelepiped of the lattice $\Omega_{k}$, where all $x_{k+1}, \ldots, x_{p}>0$, i.e. inside of $D:=\left\{x \in \mathbb{R}^{n} \mid 0<x_{i}<m_{i} \quad(i=1, \ldots k)\right.$ and $\left.x_{j}>0 \quad(k+1<j<p)\right\}$. Strongly Lipschitz means that locally the hypersurface is the graph of a Lipschitz function, and that globally the local Lipschitz constants are bounded. Suppose also that $V$ is a domain lying in $D$ and that $S$ bounds a subdomain $W$ of $V$ such that $W \cup S \subset V$. Now take an element $y \in W$. As a consequence of the usual Cauchy formula and Cauchy's theorem in $\mathbb{R}^{n}$, we obtain that if $f: V \rightarrow C l_{n}$ a left parabolic monogenic function, then

$$
\begin{aligned}
f(y, t)= & \int_{S}\left(\sum_{\varepsilon_{k+1}, \ldots, \varepsilon_{p} \in\{ \pm 1\}} \sum_{\underline{\omega} \in \Omega_{k}} G\left(\underline{x}-\underline{y}+\underline{\omega}, \varepsilon_{k+1}\left(x_{k+1}-y_{k+1}\right), \ldots, \varepsilon_{p}\left(x_{p}-y_{p}\right),\left(x_{p+1}-y_{p+1}\right), \ldots,\left(x_{n}-y_{n}\right), t\right)\right) \\
& \times n(x, t) f(x, t) d \sigma(x, t),
\end{aligned}
$$

where $n(x, t)$ stands for the unit exterior normal vector to $S$ at $(x, t)$ and $\sigma$ denotes the usual Lebesgue measure on $S$. As $S, V, W$ entirely lie in $D$, the projection map $p_{k}$ induces a Lipschitz surface $S^{\prime}$, a domain $V^{\prime}$ and a subdomain $W^{\prime}$ of $V^{\prime}$ that lie entirely on that part of the infinite cylinder $C_{k}$ where additionally $x_{k+1}, \ldots, x_{p}>0$. So, the original sets $V, W$ descend to well-defined domains $V^{\prime \prime}, W^{\prime \prime}$ and the surface $S$ to a well-defined strongly Lipschitz hypersurface $S^{\prime \prime}$ on the projective cylinder $\mathcal{M}_{k, p}$. As a consequence of the usual Cauchy's theorem from [12] we can now formulate

Theorem 4.2 (Cauchy integral formula) Suppose that $S^{\prime \prime}$ is strongly Lipschitz hyperface bounding a subdomain $W^{\prime \prime}$ of a domain $V^{\prime \prime}$ lying on the orbifold $\mathcal{M}_{k, p}$. Let $f^{\prime \prime}: V^{\prime \prime} \rightarrow E^{1}$ be a left parabolic monogenic section on $\mathcal{M}_{k, p}$ with values in the trivial pin bundle $E^{1}$. Suppose that $y^{\prime \prime} \in W^{\prime \prime}$. Then

$$
f^{\prime \prime}\left(y^{\prime \prime}, t\right)=\int_{S^{\prime \prime}} G_{\mathcal{M}_{k, p}}\left(x^{\prime \prime}, y^{\prime \prime}, t\right) d P_{k, p}(n(x, t)) f^{\prime \prime}\left(x^{\prime \prime}, t\right) d \sigma^{\prime \prime}\left(x^{\prime \prime}, t\right),
$$

where $d P_{k, p}$ stands for the derivative of the projection map $P_{k, p}=P_{p} \circ p_{k}$. 
In view of the well-known Almansi-Fischer decomposition theorem we can now directly set up a Green's integral formula for sections in the kernel of the heat operator on these orbifolds. To proceed in this direction, we need to construct the Green's kernel for harmonic sections on $\mathcal{M}_{k, p}$. We can obtain the Green's kernel on $\mathcal{M}_{k, p}$ as a finite sum of harmonic Green's kernels that we constructed for the fully infinite cylinders $C_{k}$. By similar arguments as given above the harmonic Green's kernel for harmonic sections on $\mathcal{M}_{k, p}$ with values in the trivial pin bundle $E^{1}$ can be expressed by

$H_{\mathcal{M}_{k, p}}\left(x^{\prime \prime}, y^{\prime \prime}, t\right)=P_{p}\left(\sum_{\varepsilon_{k+1}, \ldots, \varepsilon_{p} \in\{ \pm 1\}} H_{k}\left(\underline{x^{\prime}}-\underline{y^{\prime}}, \varepsilon_{k+1}\left(x_{k+1}^{\prime}-y_{k+1}^{\prime}\right), \ldots, \varepsilon_{p}\left(x_{p}-y_{p}\right),\left(x_{p+1}^{\prime}-y_{p+1}^{\prime}\right), \ldots\left(x_{n}^{\prime}-y_{n}^{\prime}\right), t\right)\right)$

where $H_{k}\left(x^{\prime}, y^{\prime}, t\right)$ is the harmonic Green's kernel for harmonic sections on $C_{k}$ with values in the trivial pin bundle. This tool in hand, we can establish, after having applied the Almansi-Fischer decomposition, the following

Theorem 4.3 (Green's integral formula) Let $k<n-1$. Suppose that $V^{\prime \prime}, W^{\prime \prime}, S^{\prime \prime}$ and $y^{\prime \prime}$ are as in Theorem 4.2. Now let $f^{\prime \prime}: V^{\prime \prime} \rightarrow E^{1}$ be a harmonic section on $\mathcal{M}_{k, p}$. Then

$$
\begin{aligned}
f^{\prime \prime}\left(y^{\prime \prime}, t\right)= & \int_{S^{\prime \prime}} G_{\mathcal{M}_{k, p}}\left(x^{\prime \prime}, y^{\prime \prime}, t\right) d P_{k, p}(n(x, t)) f^{\prime \prime}\left(x^{\prime \prime}, t\right) d \sigma^{\prime \prime}\left(x^{\prime \prime}, t\right) \\
& +\int_{S^{\prime \prime}} H_{\mathcal{M}_{k, p}}\left(x^{\prime \prime}, y^{\prime \prime}, t\right) d P_{k, p}(n(x, t)) D^{\prime \prime}\left[f^{\prime \prime}\left(x^{\prime \prime}, t\right)\right] d \sigma^{\prime \prime}\left(x^{\prime \prime}\right)
\end{aligned}
$$

where $D^{\prime \prime}$ denotes the parabolic Dirac operator on $\mathcal{M}_{k, p}$ induced by the projection $P_{k, p}$.

Here, we see that the knowledge of the Cauchy kernel for the Dirac operator is indeed useful to study harmonic sections on these orbifolds. In the cases $k=n-1, k=n$ we can also set up a local version of the Green's integral formula, which however then involves a Cauchy kernel with more than one singularity on the whole orbifold $\mathcal{M}_{k, p}$, similarly to that on the $n-1$-cylinders and $n$-tori discussed in [16].

Remark 4.4 In the extreme case where one puts $k=0$ in which we identify the pair

$$
\left(x_{1}, \ldots, x_{n}, X, t\right)
$$

with

$$
\left(-x_{1}, \ldots,-x_{p}, x_{p+1}, \ldots, x_{n}, X, t\right)
$$

we are dealing with real projective manifolds without periodicity conditions. The latter shall be denoted by $\mathcal{M}_{0, p}$. In this limit case we simply have to replace the function

$$
G_{k}\left(\underline{x}-\underline{y}, \varepsilon_{k+1}\left(x_{k+1}-y_{k+1}\right), \ldots, \varepsilon_{p}\left(x_{p}-y_{p}\right),\left(x_{p+1}-y_{p+1}\right), \ldots,\left(x_{n}-y_{n}\right), t\right)
$$

by the function

$$
G\left(\varepsilon_{1}\left(x_{1}-y_{1}\right), \ldots, \varepsilon_{p}\left(x_{p}-y_{p}\right),\left(x_{p+1}-y_{p+1}\right), \ldots,\left(x_{n}-y_{n}\right), t\right)
$$

and the Cauchy kernel to the parabolic Dirac operator on $\mathcal{M}_{0, p}$ is simply given by

$$
\sum_{\varepsilon_{1}, \ldots, \varepsilon_{p} \in\{ \pm 1\}} G\left(\varepsilon_{1}\left(x_{1}-y_{1}\right), \ldots, \varepsilon_{p}\left(x_{p}-y_{p}\right),\left(x_{p+1}-y_{p+1}\right), \ldots,\left(x_{n}-y_{n}\right), t\right) .
$$

Note that this is only a finite series. Similarly, one constructs the corresponding harmonic kernel for the heat operator.

\section{The case of the Schrödinger operator}

\subsection{The inhomogeneous regularized Schrödinger equation on orbifolds}

In this section we will concentrate ourselves on the backward case of the Schrödinger operator, i.e., $-\Delta-i \partial_{t}$. Throughout this section, we will consider $S, V, W, S^{\prime}, V^{\prime}, W^{\prime}, S^{\prime \prime}, V^{\prime \prime}$ and $W^{\prime \prime}$ under the same conditions as in the preceding sections. However, we additionally suppose that $f: W^{\prime \prime} \rightarrow \mathbb{C}_{n}$ is a functional belonging to the Sobolev space $W_{p}^{2}\left(W^{\prime \prime}\right)$ with $1<p<\infty$. Consider also $\left(\Delta_{x^{\prime \prime}}-\mathbf{k} \partial_{t}\right)^{\prime \prime}$ as the regularized Schrödinger operator on $\mathcal{M}_{k, p}$ induced by the projection $P_{k, p}$. As it was done in [17] we can present an analogue of the Borel-Pompeiu formula (8) to the present setting: 
Theorem 5.1 Consider $V^{\prime \prime}$ under the same conditions as mentioned Theorem 4.2. Suppose that $\theta: \operatorname{cl}\left(V^{\prime \prime}\right) \rightarrow$ $\mathbb{C}_{n}$ is a continuous function such that $\left.\theta\right|_{V^{\prime \prime}}$ belongs to $C^{1}\left(V^{\prime \prime}\right)$. Then, for each pair $\left(y^{\prime \prime}, t\right) \in V^{\prime \prime}$

$$
\begin{aligned}
\theta\left(y^{\prime \prime}, t_{0}\right)= & \int_{\partial V^{\prime \prime}} G_{-, \mathcal{M}_{k, p}}^{\epsilon}\left(x^{\prime \prime}, y^{\prime \prime}, t\right)\left(d_{x} P_{k, p} n(x, t)\right) \theta\left(x^{\prime \prime}, t\right) d S\left(x^{\prime \prime}, t\right) \\
& -\int_{V^{\prime \prime}} G_{\mathcal{M}_{k, p}}^{\epsilon}\left(x^{\prime \prime}, y^{\prime \prime}, t\right)\left(D_{-}^{\epsilon}\right)^{\prime \prime} \theta\left(x^{\prime \prime}, t\right) d \mu\left(x^{\prime \prime}, t\right)
\end{aligned}
$$

where we consider the regularized fundamental solution $E_{-}^{\epsilon}$ in the construction of $G_{-, \mathcal{M}_{k, p}}^{\epsilon}$.

From the previous theorem we obtain

Definition 5.2 The regularized Cauchy operator on the above introduced orbifolds is defined from $W_{p}^{l}\left(V^{\prime \prime}\right)$ to $W_{p+1}^{l}\left(V^{\prime \prime}\right)$ with $1<p<\infty$ and has the form

$$
\left[T_{-, \mathcal{M}_{k, p}}^{\epsilon} f^{\prime \prime}\left(y^{\prime \prime}, t_{0}\right)\right]=-\int_{V^{\prime \prime}} G_{-, \mathcal{M}_{k, p}}^{\epsilon}\left(x^{\prime \prime}, y^{\prime \prime}, t\right) f^{\prime \prime}\left(x^{\prime \prime}, t\right) d V^{\prime \prime}\left(x^{\prime \prime}\right) d t
$$

where $x^{\prime \prime}$ and $y^{\prime \prime}$ are distinct points from $V^{\prime \prime}$.

Due to the exponential decrease of the kernel function, the Teodorescu transform is always an $L_{2}$ bounded operator even if $V^{\prime \prime}$ is an unbounded domain. Theorem 5.1 also allows us to introduce

Definition 5.3 The regularized Cauchy operator on the above introduced orbifolds is defined from $W_{p-1}^{l}\left(\partial V^{\prime \prime}\right)$ to $W_{p}^{l}\left(V^{\prime \prime}\right) \cap \operatorname{Ker}\left(\left(D_{-}^{\epsilon}\right)^{\prime \prime}\right)$ with $1<p<\infty$ and has the form

$$
\left[F_{-, \mathcal{M}_{k, p}}^{\epsilon} f^{\prime \prime}\left(y^{\prime \prime}, t_{0}\right)\right]=-\int_{\partial V^{\prime \prime}} G_{-, \mathcal{M}_{k, p}}^{\epsilon}\left(x^{\prime \prime}, y^{\prime \prime}, t\right) n\left(x^{\prime \prime}, t\right)\left(d_{x} P_{k, p} n(x, t)\right) f^{\prime \prime}\left(x^{\prime \prime}, t\right) d S^{\prime \prime}\left(x^{\prime \prime}, t\right) .
$$

Using the previously introduced operators, the Borel-Pompeiu formula presented in Theorem 5.1 can now be reformulated in the classical form

$$
f^{\prime \prime}=F_{-, \mathcal{M}_{k, p}}^{\epsilon} f^{\prime \prime}+T_{-, \mathcal{M}_{k, p}}^{\epsilon}\left(D_{-}^{\epsilon}\right)^{\prime \prime} f^{\prime \prime},
$$

as it has previously been formulated for the Euclidian case (see [5, 13]) in the context of elliptic operators. Adapting the arguments from $[5,17]$ that were explicitly worked out for the Euclidian case, one can show that the following Hodge-type decomposition holds for the space of the $L_{p}$ functions over a domain $V^{\prime \prime}$ of the orbifold $\mathcal{M}_{k, p}$.

Theorem 5.4 The space $L_{p}\left(V^{\prime \prime}\right), 1<p<\infty$ admits the following decomposition

$$
L_{p}\left(V^{\prime \prime}\right)=\left(L_{p}\left(V^{\prime \prime}\right) \cap \operatorname{Ker}\left(\left(D_{-}^{\epsilon}\right)^{\prime \prime}\right)\right) \oplus\left(D_{-}^{\epsilon}\right)^{\prime \prime}\left(\stackrel{\circ}{\left.\left.W_{p}^{1} V^{\prime \prime}\right)\right)}\right.
$$

for all $\epsilon>0$. Furthermore, we can define the following projectors

$$
P_{-, \mathcal{M}_{k, p}}^{\epsilon}: L_{p}\left(V^{\prime \prime}\right) \rightarrow \operatorname{Ker}\left(\left(D_{-}^{\epsilon}\right)^{\prime \prime}\right) \cap L_{p}\left(V^{\prime \prime}\right) \quad Q_{-, \mathcal{M}_{k, p}}^{\epsilon} \quad L_{p}\left(V^{\prime \prime}\right) \rightarrow\left(D_{-}^{\epsilon}\right)^{\prime \prime}\left({ }^{\circ} W_{p}^{1}\left(V^{\prime \prime}\right)\right)
$$

where $P_{-, \mathcal{M}_{k, p}}^{\epsilon}$ corresponds to the regularized Bergman projector on these orbifolds and $Q_{-, \mathcal{M}_{k, p}}^{\epsilon}=I-P_{-, \mathcal{M}_{k, p}}^{\epsilon}$ is called regularized Pompeiu projector in the context of these orbifolds.

For the particular case $p=2$ this decomposition is orthogonal and the space $\operatorname{Ker}\left(\left(D_{-}^{\epsilon}\right)^{\prime \prime}\right) \cap L_{2}\left(V^{\prime \prime}\right)$ is a Banach space endowed with the $L_{2}$ inner product

$$
\left\langle f^{\prime}, g^{\prime}\right\rangle:=\int_{V^{\prime \prime}} \overline{f\left(x^{\prime \prime}, t\right)} g\left(x^{\prime \prime}, t\right) d V\left(x^{\prime \prime}\right) d t
$$

Then, as a consequence of Cauchy's integral formula and Cauchy-Schwarz' equality we can show that this space has a continuous point evaluation and does hence possess a reproducing kernel $B\left(x^{\prime}, y^{\prime}, t\right)$, satisfying

$$
f^{\prime \prime}\left(y^{\prime \prime}, t_{0}\right)=\int_{V^{\prime \prime}} B\left(x^{\prime \prime}, y^{\prime \prime}, t\right) f\left(x^{\prime \prime}, t\right) d V\left(x^{\prime \prime}\right) d t, \quad \forall f^{\prime \prime} \in \operatorname{Ker}\left(\left(D_{-}^{\epsilon}\right)^{\prime \prime}\right) \cap L_{2}\left(V^{\prime \prime}\right) .
$$


Let $f$ be an arbitrary function from $L_{2}\left(V^{\prime \prime}\right)$. Then the operator

$$
\left[P_{-, \mathcal{M}_{k, p}}^{\epsilon} f^{\prime \prime}\left(y^{\prime \prime}, t\right)\right]=\int_{V^{\prime \prime}} B\left(x^{\prime \prime}, y^{\prime \prime}, t\right) f\left(x^{\prime \prime}, t\right) d V\left(x^{\prime \prime}\right) d t
$$

corresponds to the projector presented in Theorem 5.4 for $p=2$. With these operators we can represent the solutions to the inhomogeneous regularized Schrödinger equation in orbifolds, in complete analogy to the Euclidean case treated in [7]. We establish

Theorem 5.5 Let $V^{\prime \prime}$ be a domain on the orbifold $\mathcal{M}_{k, p}$ and $f \in W_{p}^{2}\left(V^{\prime \prime}\right)$ with $1<p<\infty$. Then the system

$$
\begin{aligned}
\left(-\Delta_{x^{\prime \prime}}-\mathbf{k} \partial_{t}\right)^{\prime \prime} u^{\prime \prime} & =f^{\prime \prime} \text { in } V^{\prime \prime} \\
u^{\prime \prime} & =0 \text { at } \partial V^{\prime \prime}
\end{aligned}
$$

has a unique solution $u \in W_{p+2, l o c}^{2}\left(V^{\prime \prime}\right)$ of the form

$$
u^{\prime \prime}=T_{-, \mathcal{M}_{k, p}}^{\epsilon} Q_{-, \mathcal{M}_{k, p}}^{\epsilon} T_{-, \mathcal{M}_{k, p}}^{\epsilon} f^{\prime \prime} .
$$

Proof: To the proof one applies the factorization $\left(\left(D_{-}^{\epsilon}\right)^{\prime \prime}\right)^{2}=\left(-\Delta_{x^{\prime \prime}}-\mathbf{k} \partial_{t}\right)^{\prime \prime}$. Thus, equation (11) can be rewritten in the form

$$
\left(\left(D_{-}^{\epsilon}\right)^{\prime \prime}\right)^{2} u^{\prime \prime}=f^{\prime \prime}
$$

Now one applies the regularized Teodorescu transform on the orbifolds $T_{-, \mathcal{M}_{k, p}}^{\epsilon}$ to this equation which leads to

$$
T_{-, \mathcal{M}_{k, p}}^{\epsilon}\left(D_{-}^{\epsilon}\right)^{\prime \prime}\left[\left(D_{-}^{\epsilon}\right)^{\prime \prime} u^{\prime \prime}\right]=T_{-, \mathcal{M}_{k, p}}^{\epsilon} f^{\prime \prime} .
$$

Next we apply the generalized Borel-Pompeiu's formula in the setting of these orbifolds which leads to

$$
\left(D_{-}^{\epsilon}\right)^{\prime \prime} u^{\prime \prime}-F_{-, \mathcal{M}_{k, p}}^{\epsilon}\left(D_{-}^{\epsilon}\right)^{\prime \prime} u^{\prime \prime}=T_{-, \mathcal{M}_{k, p}}^{\epsilon} f^{\prime \prime} .
$$

Applying the projector $Q_{-, \mathcal{M}_{k, p}}^{\epsilon}$ to the previous equation leads to

$$
Q_{-, \mathcal{M}_{k, p}}^{\epsilon}\left(D_{-}^{\epsilon}\right)^{\prime \prime} u^{\prime \prime}-Q_{-, \mathcal{M}_{k, p}}^{\epsilon} F_{-, \mathcal{M}_{k, p}}^{\epsilon}\left(D_{-}^{\epsilon}\right)^{\prime \prime} u^{\prime \prime}=Q_{-, \mathcal{M}_{k, p}}^{\epsilon} T_{-, \mathcal{M}_{k, p}}^{\epsilon} f^{\prime \prime} .
$$

Since $F_{-, \mathcal{M}_{k, p}}^{\epsilon}\left(D_{-}^{\epsilon}\right)^{\prime \prime} u^{\prime \prime} \in \operatorname{Ker}\left(\left(D_{-}^{\epsilon}\right)^{\prime \prime}\right)$ one has $Q_{-, \mathcal{M}_{k, p}}^{\epsilon} F_{-, \mathcal{M}_{k, p}}^{\epsilon}\left(D_{-}^{\epsilon}\right)^{\prime \prime} u^{\prime \prime}=0$. Therefore, equation (15) is equivalent to

$$
Q_{-, \mathcal{M}_{k, p}}^{\epsilon}\left(D_{-}^{\epsilon}\right)^{\prime \prime} u^{\prime \prime}=Q_{-, \mathcal{M}_{k, p}}^{\epsilon} T_{-, \mathcal{M}_{k, p}}^{\epsilon} f^{\prime \prime} .
$$

Next, we again apply the regularized Teodorescu transform in the context of these orbifolds to this equation. This leads to

$$
T_{-, \mathcal{M}_{k, p}}^{\epsilon} Q_{-, \mathcal{M}_{k, p}}^{\epsilon}\left(D_{-}^{\epsilon}\right)^{\prime \prime} u^{\prime \prime}=T_{-, \mathcal{M}_{k, p}}^{\epsilon} Q_{-, \mathcal{M}_{k, p}}^{\epsilon} T_{-, \mathcal{M}_{k, p}}^{\epsilon} f^{\prime \prime}
$$

Applying the specific mapping properties of these operators and again Borel-Pompeiu's formula, then the left hand-side of this equation simplifies to $u^{\prime \prime}$ so that we finally obtain that

$$
u^{\prime \prime}=T_{-, \mathcal{M}_{k, p}}^{\epsilon} Q_{-, \mathcal{M}_{k, p}}^{\epsilon} T_{-, \mathcal{M}_{k, p}}^{\epsilon} f^{\prime \prime} .
$$

The assertion now follows.

By adapting the standard techniques from [13] to the setting of this paper we have the following generalization of the previous result

Theorem 5.6 Let $V^{\prime \prime}$ be a domain on the orbifold $\mathcal{M}_{k, p}, f^{\prime \prime} \in W_{p}^{2}\left(V^{\prime \prime}\right)$ and $g^{\prime \prime} \in W_{p+3 / 2}^{2}\left(\partial V^{\prime \prime}\right)$ with $1<p<\infty$. Then the system

$$
\begin{aligned}
\left(\Delta_{x^{\prime \prime}}-\mathbf{k} \partial_{t}\right)^{\prime \prime} u^{\prime \prime} & =f^{\prime \prime} \text { in } V^{\prime \prime} \\
u^{\prime \prime} & =g^{\prime \prime} \text { at } \partial V^{\prime \prime}
\end{aligned}
$$

has a solution $u \in W_{p+2, \text { loc }}^{2}\left(V^{\prime \prime}\right)$ of the form

$$
u^{\prime \prime}=F_{-, \mathcal{M}_{k, p}}^{\epsilon} g^{\prime \prime}+T_{-, \mathcal{M}_{k, p}}^{\epsilon} P_{-, \mathcal{M}_{k, p}}^{\epsilon}\left(D_{-}^{\epsilon}\right)^{\prime \prime} h^{\prime \prime}+T_{-, \mathcal{M}_{k, p}}^{\epsilon} Q_{-, \mathcal{M}_{k, p}}^{\epsilon} T_{-, \mathcal{M}_{k, p}}^{\epsilon} f^{\prime \prime},
$$

where $h^{\prime \prime}$ is the unique $W_{p+2}^{2}\left(V^{\prime \prime}\right)$ extension of $g^{\prime \prime}$. 
Remark 5.7 Again, as in [13] we can represent the regularized Bergman projector on this class of orbifolds in terms of algebraic expressions involving only the regularized Cauchy and Teodorescu transform on orbifolds, viz

$$
P_{-, \mathcal{M}_{k, p}}^{\epsilon}=F_{-, \mathcal{M}_{k, p}}^{\epsilon}\left(\operatorname{tr}\left(T_{-, \mathcal{M}_{k, p}}^{\epsilon}\right) F_{-, \mathcal{M}_{k, p}}^{\epsilon}\right)^{-1} \operatorname{tr}\left(T_{-, \mathcal{M}_{k, p}}^{\epsilon}\right),
$$

where $t r$ is the usual trace operator. This formula allows us to represent the solutions to the inhomogeneous regularized Schrödinger equation on these orbifolds in terms of the singular integral operators that we introduced in the previous section.

\subsection{The limit case $\epsilon \rightarrow 0^{+}$}

The aim of this section is to extend the results presented previously to the original operators $D_{-}^{\prime \prime}$ and $\left(-\Delta_{x^{\prime \prime}}-\right.$ $\left.i \partial_{t}\right)^{\prime \prime}$. In order to proceed in this direction we start by recalling the following result from [5]

Theorem 5.8 For all $1<p<\infty$ we have the following weak convergence, in $W_{p}^{-\frac{n}{2}-1}\left(V^{\prime \prime}\right)$,

$$
\left\langle E_{+}^{\epsilon}, \varphi\right\rangle \rightarrow\left\langle E_{+}, \varphi\right\rangle, \quad \varphi \in W_{p}^{\frac{n}{2}+1}\left(V^{\prime \prime}\right),
$$

when $\epsilon \rightarrow 0$. Here $E_{+}$is given by (3) with $\alpha=i$.

This theorem implies the following corollary

Corollary 5.9 For all $1<p<\infty$ we have the following weak convergence in $W_{p}^{-\frac{n}{2}-1}\left(V^{\prime \prime}\right)$ :

$$
\left\langle G_{-, \mathcal{M}_{k, p}}^{\epsilon}, \varphi\right\rangle \rightarrow\left\langle G_{-, \mathcal{M}_{k, p}}, \varphi\right\rangle, \quad \varphi \in W_{p}^{\frac{n}{2}+1}\left(V^{\prime \prime}\right),
$$

when $\epsilon \rightarrow 0$. Here, $G_{-, \mathcal{M}_{k, p}}$ is the projection under $P_{k, p}$ of

$$
\sum_{\varepsilon_{k+1}, \ldots, \varepsilon_{p} \in\{ \pm 1\}} g_{k}\left(\underline{x^{\prime}}-\underline{y}^{\prime}, \varepsilon_{k+1}\left(x_{k+1}^{\prime}-y_{k+1}^{\prime}\right), \varepsilon_{p}\left(x_{p}^{\prime}-y_{p}^{\prime}\right),\left(x_{p+1}^{\prime}-y_{p+1}^{\prime}\right), \ldots\left(x_{n}^{\prime}-y_{n}^{\prime}\right), t\right) .
$$

On the basis of these results we are in position to study the convergence of the family of operators $T_{-, \mathcal{M}_{k, p}}^{\epsilon}$ and projectors $Q_{-, \mathcal{M}_{k, p}}^{\epsilon}$ to the Teodorescu operator and the Bergaman projector associated to the Schrödinger operator on these orbifolds defined by

$$
\begin{aligned}
& {\left[T_{-, \mathcal{M}_{k, p}} f^{\prime \prime}\left(y^{\prime \prime}, t_{0}\right)\right]=-\int_{V^{\prime \prime}} G_{-, \mathcal{M}_{k, p}}\left(x^{\prime \prime}-y^{\prime \prime}, t\right) f^{\prime \prime}\left(x^{\prime \prime}, t\right) d V^{\prime \prime}\left(x^{\prime \prime}\right) d t} \\
& Q_{-, \mathcal{M}_{k, p}}=I-P_{-, \mathcal{M}_{k, p}}=I-\int_{V^{\prime \prime}} B\left(x^{\prime \prime}, y^{\prime \prime}, t\right) f\left(x^{\prime \prime}, t\right) d V\left(x^{\prime \prime}\right) d t
\end{aligned}
$$

where $B\left(x^{\prime \prime}, y^{\prime \prime}, t\right)$ is a reproducing kernel, which satisfies

$$
f^{\prime \prime}\left(y^{\prime \prime}, t_{0}\right)=\int_{V^{\prime \prime}} B\left(x^{\prime \prime}, y^{\prime \prime}, t\right) f\left(x^{\prime \prime}, t\right) d V\left(x^{\prime \prime}\right) d t, \quad \forall f^{\prime \prime} \in \operatorname{Ker}\left(\left(D_{-}\right)^{\prime \prime}\right) \cap L_{2}\left(V^{\prime \prime}\right) .
$$

Theorem 5.10 The family of regularized Teodorescu operators on the orbifolds $T_{-, \mathcal{M}_{k, p}}$ converges weakly to $T_{-, \mathcal{M}_{k, p}}$ in $W_{p}^{\frac{n}{2}+1}\left(V^{\prime \prime}\right)$ for all $1<p<\infty$.

Proof: Let $u \in L_{p}\left(V^{\prime \prime}\right)$. From the preceding theorem we may infer that we have for every $\varphi \in W_{p}^{\frac{n}{2}+1}\left(V^{\prime \prime}\right)$,

$$
\begin{aligned}
\lim _{\epsilon \rightarrow 0^{+}}\left|\left\langle\left(T_{-, \mathcal{M}_{k, p}}^{\epsilon}-T_{-, \mathcal{M}_{k, p}}\right) u, \varphi\right\rangle\right| & =\lim _{\epsilon \rightarrow 0^{+}}\left|\left\langle\left(G_{-, \mathcal{M}_{k, p}}^{\epsilon}-G_{-, \mathcal{M}_{k, p}}\right) * u, \varphi\right\rangle\right| \\
& =\left|\left\langle\lim _{\epsilon \rightarrow 0^{+}}\left(G_{-, \mathcal{M}_{k, p}}^{\epsilon}-G_{-, \mathcal{M}_{k, p}}\right), u * \varphi\right\rangle\right| \\
& =0
\end{aligned}
$$

Theorem 5.11 The family of projectors $Q_{-, \mathcal{M}_{k, p}}^{\epsilon}$ is a fundamental family in $W_{p}^{-\frac{n}{2}-1}\left(V^{\prime \prime}\right)$ for all $1<p<\infty$. 
Proof: Let us start with the proof of the convergence. Consider $u \in L_{p}\left(V^{\prime \prime}\right)$ and $\varphi \in W_{p}^{\frac{n}{2}+1}\left(V^{\prime \prime}\right)$, where $1<p<\infty$. Since for all $\epsilon>0,\left(Q_{-, \mathcal{M}_{k, p}}^{\epsilon}\right)^{2}=Q_{-, \mathcal{M}_{k, p}}^{\epsilon}$ and $Q_{-, \mathcal{M}_{k, p}}^{\epsilon}\left(P_{-, \mathcal{M}_{k, p}}^{\epsilon} u\right)=0$, we have for any $\epsilon_{1}, \epsilon_{2}>0$

$$
\begin{aligned}
\left|\left\langle Q_{-, \mathcal{M}_{k, p}}^{\epsilon_{1}} u-Q_{-, \mathcal{M}_{k, p}}^{\epsilon_{2}} u, \varphi\right\rangle\right| & =\left|\left\langle Q_{-, \mathcal{M}_{k, p}}^{\epsilon_{1}}\left(P_{-, \mathcal{M}_{k, p}}^{\epsilon_{1}} u+Q_{-, \mathcal{M}_{k, p}}^{\epsilon_{1}} u\right)-Q_{-, \mathcal{M}_{k, p}}^{\epsilon_{2}}\left(P_{-, \mathcal{M}_{k, p}}^{\epsilon_{1}} u+Q_{-, \mathcal{M}_{k, p}}^{\epsilon_{1}} u\right), \varphi\right\rangle\right| \\
& =\left|\left\langle Q_{-, \mathcal{M}_{k, p}}^{\epsilon_{1}} u-Q_{-, \mathcal{M}_{k, p}}^{\epsilon_{2}} P_{-, \mathcal{M}_{k, p}}^{\epsilon_{2}} u-Q_{-, \mathcal{M}_{k, p}}^{\epsilon_{2}} Q_{-, \mathcal{M}_{k, p}}^{\epsilon_{1}} u, \varphi\right\rangle\right| \\
& \leq \underbrace{\left|\left\langle Q_{-, \mathcal{M}_{k, p}}^{\epsilon_{2}} P_{-, \mathcal{M}_{k, p}}^{\epsilon_{1}} u, \varphi\right\rangle\right|}_{(A)}+\underbrace{\left|\left\langle\left(I-Q_{-, \mathcal{M}_{k, p}}^{\epsilon_{2}}\right) Q_{-, \mathcal{M}_{k, p}}^{\epsilon_{1}} u, \varphi\right\rangle\right|}_{(A)} .
\end{aligned}
$$

For $P_{-, \mathcal{M}_{k, p}}^{\epsilon}: L_{p}\left(V^{\prime \prime}\right) \rightarrow \operatorname{Ker}\left(\left(D_{-}^{\epsilon}\right)^{\prime \prime}\right) \cap L_{p}\left(V^{\prime \prime}\right)$ the projectors defined previously, we have for the term $(A)$

$$
\begin{aligned}
\left|\left\langle Q_{-, \mathcal{M}_{k, p}}^{\epsilon_{2}} P_{-, \mathcal{M}_{k, p}}^{\epsilon_{1}} u, \varphi\right\rangle\right| & =\left|\left\langle Q_{-, \mathcal{M}_{k, p}}^{\epsilon_{2}}\left(F_{-, \mathcal{M}_{k, p}}^{\epsilon_{1}} P_{-, \mathcal{M}_{k, p}}^{\epsilon_{1}}-Q_{-, \mathcal{M}_{k, p}}^{\epsilon_{2}} F_{-, \mathcal{M}_{k, p}}^{\epsilon_{2}}\right) P_{-, \mathcal{M}_{k, p}}^{\epsilon_{1}} u, \varphi\right\rangle\right| \\
& =\left|\left\langle Q_{-, \mathcal{M}_{k, p}}^{\epsilon_{2}}\left(I-T_{-, \mathcal{M}_{k, p}}^{\epsilon_{1}}\left(D_{-}^{\epsilon_{1}}\right)^{\prime \prime}-\left(I-T_{-, \mathcal{M}_{k, p}}^{\epsilon_{2}}\left(D_{-}^{\epsilon_{2}}\right)^{\prime \prime}\right)\right) P_{-, \mathcal{M}_{k, p}}^{\epsilon_{1}} u, \varphi\right\rangle\right| \\
& =\left|\left\langle Q_{-, \mathcal{M}_{k, p}}^{\epsilon_{2}}\left(T_{-, \mathcal{M}_{k, p}}^{\epsilon_{1}}\left(D_{-}^{\epsilon_{1}}\right)^{\prime \prime}-T_{-, \mathcal{M}_{k, p}}^{\epsilon_{2}}\left(D_{-}^{\epsilon_{2}}\right)^{\prime \prime}\right) P_{-, \mathcal{M}_{k, p}}^{\epsilon_{1}} u, \varphi\right\rangle\right| \\
& =\left|\left\langle Q_{-, \mathcal{M}_{k, p}}^{\epsilon_{2}}\left(T_{-, \mathcal{M}_{k, p}}^{\epsilon_{1}}\left(\left(D_{-}^{\epsilon_{1}}\right)^{\prime \prime}-\left(D_{-}^{\epsilon_{2}}\right)^{\prime \prime}\right)+\left(T_{-, \mathcal{M}_{k, p}}^{\epsilon_{1}}-T_{-, \mathcal{M}_{k, p}}^{\epsilon_{2}}\right)\left(D_{-}^{\epsilon_{2}}\right)^{\prime \prime}\right) P_{-, \mathcal{M}_{k, p}}^{\epsilon_{1}} u, \varphi\right\rangle\right| .
\end{aligned}
$$

From Theorem 2.3 and Theorem 5.10 we may deduce the weak convergence of $(A)$, in $W_{p}^{-\frac{n}{2}-1}\left(V^{\prime \prime}\right)$ for all $1<p<\infty$, of the right hand side of the last expression to zero. Finally, since $Q_{-, \mathcal{M}_{k, p}}^{\epsilon_{1}} u \in\left(D_{-}\right)^{\prime \prime}\left(\stackrel{\circ}{W_{p}^{1}}\left(V^{\prime \prime}\right)\right)$, there exists $g \in \stackrel{\circ}{W_{p}^{1}}\left(V^{\prime \prime}\right)$ such that $u=\left(D_{-}^{\epsilon}\right)^{\prime \prime} g$. Therefore, $(B)$ becomes

$$
\begin{aligned}
\left|\left\langle\left(I-Q_{-, \mathcal{M}_{k, p}}^{\epsilon_{2}}\right) Q_{-, \mathcal{M}_{k, p}}^{\epsilon_{1}} u, \varphi\right\rangle\right| & =\left|\left\langle\left(I-Q_{-, \mathcal{M}_{k, p}}^{\epsilon_{2}}\right)\left(D_{-}^{\epsilon}\right)^{\prime \prime} g, \varphi\right\rangle\right| \\
& =\left|\left\langle\left(D_{-}^{\epsilon_{1}}\right)^{\prime \prime} g-Q_{-, \mathcal{M}_{k, p}}^{\epsilon_{2}}\left(D_{-}^{\epsilon_{1}}\right)^{\prime \prime} g+\left(D_{-}^{\epsilon_{2}}\right)^{\prime \prime} g-\left(D_{-}^{\epsilon_{2}}\right)^{\prime \prime} g \varphi\right\rangle\right| \\
& =\left|\left\langle Q_{-, \mathcal{M}_{k, p}}^{\epsilon_{2}}\left(\left(D_{-}^{\epsilon}\right)^{\prime \prime} g-\left(D_{-}\right)^{\prime \prime} g\right)+\left(\left(D_{-}\right)^{\prime \prime} g-\left(D_{-}^{\epsilon}\right)^{\prime \prime} g\right), \varphi\right\rangle\right| \\
& =\left|\left\langle\left(\left(D_{-}\right)^{\prime \prime} g-\left(D_{-}^{\epsilon}\right)^{\prime \prime} g\right)\left(I-Q_{-, \mathcal{M}_{k, p}}^{\epsilon_{1}}\right), \varphi\right\rangle\right| .
\end{aligned}
$$

Once again, by Theorem 2.3 we conclude that the latter expression tends to zero when $\epsilon \rightarrow 0$.

Now it remains to prove that $Q_{-, \mathcal{M}_{k, p}}$ is idempotent. Hereby, we have

$$
\left(Q_{-, \mathcal{M}_{k, p}}\right)^{2}=\lim _{\epsilon \rightarrow 0}\left(Q_{-, \mathcal{M}_{k, p}}^{\epsilon}\right)^{2}=\lim _{\epsilon \rightarrow 0} Q_{-, \mathcal{M}_{k, p}}^{\epsilon}=Q_{-, \mathcal{M}_{k, p}} .
$$

Theorem 5.12 For any given $f \in L_{p}\left(V^{\prime \prime}\right)$, consider the solutions $\left(u^{\epsilon}\right)$ for the problem

$$
\begin{aligned}
\left(-\Delta_{x^{\prime \prime}}-\mathbf{k} \partial_{t}\right)^{\prime \prime}\left(u^{\epsilon}\right)^{\prime \prime} & =f^{\prime \prime} \text { in } V^{\prime \prime} \\
\left(u^{\epsilon}\right)^{\prime \prime} & =0 \quad \text { at } \partial V^{\prime \prime \prime}
\end{aligned}
$$

for each $\epsilon>0$. Then, the family of those solutions $\left(\left(u^{\epsilon}\right)^{\prime \prime}\right)$ is a fundamental family in $W_{p}^{-\frac{n}{p}-1}\left(V^{\prime \prime}\right)$, for all $1<p<\infty$. Moreover, $\left(\left(D_{-}^{\epsilon}\right)^{\prime \prime}\left(u^{\epsilon}\right)^{\prime \prime}\right)$ is a fundamental family in $W_{p}^{-\frac{n}{p}-1}\left(V^{\prime \prime}\right)$.

Proof: Let us consider $\varphi \in W_{p}^{\frac{n}{2}+1}\left(V^{\prime \prime}\right), f \in L_{p}\left(V^{\prime \prime}\right)$ and a family of functions $\left(\left(u^{\epsilon}\right)^{\prime \prime}\right)$, such that $\left(u^{\epsilon}\right)^{\prime \prime} \in$ $\left(D_{-}^{\epsilon}\right)^{\prime \prime}\left(V^{\prime \prime}\right)$ with $\epsilon>0$, and $\epsilon_{1}, \epsilon_{2}>0$. Since the elements of the family are solutions of the problem, we have that $\left(u^{\epsilon}\right)^{\prime \prime}=T_{-, \mathcal{M}_{k, p}}^{\epsilon} Q_{-, \mathcal{M}_{k, p}}^{\epsilon} T_{-, \mathcal{M}_{k, p}}^{\epsilon} f$ (for more details about this assertion see [5, 17]). Then

$$
\begin{aligned}
\left|\left\langle\left(u^{\epsilon_{1}}\right)^{\prime \prime}-\left(u^{\epsilon_{2}}\right)^{\prime \prime}, \varphi\right\rangle\right|= & \left|\left\langle T_{-, \mathcal{M}_{k, p}}^{\epsilon_{1}} Q_{-, \mathcal{M}_{k, p}}^{\epsilon_{1}} T_{-, \mathcal{M}_{k, p}}^{\epsilon_{1}} f-T_{-, \mathcal{M}_{k, p}}^{\epsilon_{2}} Q_{-, \mathcal{M}_{k, p}}^{\epsilon_{2}} T_{-, \mathcal{M}_{k, p}}^{\epsilon_{2}} f, \varphi\right\rangle\right| \\
= & \left|\left\langle\left(T_{-, \mathcal{M}_{k, p}}^{\epsilon_{1}} Q_{-, \mathcal{M}_{k, p}}^{\epsilon_{1}} T_{-, \mathcal{M}_{k, p}}^{\epsilon_{1}}-T_{-, \mathcal{M}_{k, p}}^{\epsilon_{2}} Q_{-, \mathcal{M}_{k, p}}^{\epsilon_{2}} T_{-, \mathcal{M}_{k, p}}^{\epsilon_{2}}\right) f, \varphi\right\rangle\right| \\
\leq & \left|\left\langle\left(T_{-, \mathcal{M}_{k, p}}^{\epsilon_{1}} Q_{-, \mathcal{M}_{k, p}}^{\epsilon_{1}}\left(T_{-, \mathcal{M}_{k, p}}^{\epsilon_{1}}-T_{-, \mathcal{M}_{k, p}}^{\epsilon_{2}}\right)\right) f, \varphi\right\rangle\right| \\
& +\left|\left\langle\left(\left(T_{-, \mathcal{M}_{k, p}}^{\epsilon_{1}}-T_{-, \mathcal{M}_{k, p}}^{\epsilon_{2}}\right) Q_{-}^{C_{k}, \epsilon_{1}} T_{-, \mathcal{M}_{k, p}}^{\epsilon_{2}}\right) f, \varphi\right\rangle\right| \\
& +\left|\left\langle\left(T_{-, \mathcal{M}_{k, p}}^{\epsilon_{1}}\left(Q_{-, \mathcal{M}_{k, p}}^{\epsilon_{1}}-Q_{-, \mathcal{M}_{k, p}}^{\epsilon_{\epsilon}}\right) T_{-, \mathcal{M}_{k, p}}^{\epsilon_{2}}\right) f, \varphi\right\rangle\right| .
\end{aligned}
$$


By Theorem 5.10 and Theorem 5.11 we conclude that the right hand side of the last inequality tends to zero when $\epsilon_{1}, \epsilon_{2} \rightarrow 0$. Theorem 5.12 now guarantees that there exists a function $f \in L_{p}\left(V^{\prime \prime}\right)$ such that

$$
\left(D_{-}^{\epsilon_{1}}\right)^{\prime \prime}\left(u^{\epsilon_{1}}\right)^{\prime \prime}=Q_{-, \mathcal{M}_{k, p}}^{\epsilon_{1}} T_{-, \mathcal{M}_{k, p}}^{\epsilon_{1}} f \quad \text { and } \quad\left(D_{-}^{\epsilon_{2}}\right)^{\prime \prime}\left(u^{\epsilon_{2}}\right)^{\prime \prime}=Q_{-, \mathcal{M}_{k, p}}^{\epsilon_{2}} T_{-, \mathcal{M}_{k, p}}^{\epsilon_{2}} f .
$$

This in turn implies that

$$
\begin{aligned}
& \left|\left\langle\left(Q_{-, \mathcal{M}_{k, p}}^{\epsilon_{1}} T_{-, \mathcal{M}_{k, p}}^{\epsilon_{1}}-Q_{-, \mathcal{M}_{k, p}}^{\epsilon_{2}} T_{-, \mathcal{M}_{k, p}}^{\epsilon_{2}}\right) f, \varphi\right\rangle\right| \\
& \quad \leq\left|\left\langle\left(Q_{-, \mathcal{M}_{k, p}}^{\epsilon_{1}}\left(T_{-, \mathcal{M}_{k, p}}^{\epsilon_{1}}-T_{-, \mathcal{M}_{k, p}}^{\epsilon_{2}}\right)\right) f, \varphi\right\rangle\right|+\left|\left\langle\left(\left(Q_{-, \mathcal{M}_{k, p}}^{\epsilon_{1}}-Q_{-, \mathcal{M}_{k, p}}^{\epsilon_{2}}\right) T_{-, \mathcal{M}_{k, p}}^{\epsilon_{2}}\right) f, \varphi\right\rangle\right| .
\end{aligned}
$$

By Theorem 5.11 and Theorem 5.12 we conclude that the right hand side of the previous expression converges weakly to zero when $\left|\epsilon_{1}-\epsilon_{2}\right| \rightarrow 0$, in $W_{p}^{-\frac{n}{2}-1}\left(V^{\prime \prime}\right)$ for all $1<p<\infty$.

This result can be refined. By $\left(u_{2}\right)^{\prime \prime} \in W_{p}^{-\frac{n}{2}-1}\left(V^{\prime \prime}\right)$ we denote the function limit of the Cauchy family that we studied. Again Theorem 5.12 implies the existence of functions $f \in L_{p}\left(V^{\prime \prime}\right)$ that satisfy

$$
\left(-\Delta-i \partial_{t}\right)^{\prime \prime}\left(u_{2}\right)^{\prime \prime}=f \quad \text { and } \quad\left(-\Delta-i \partial_{t}\right)^{\prime \prime}\left(u_{2}^{\epsilon}\right)^{\prime \prime}=f
$$

with $\left.\left(u_{2}\right)^{\prime \prime}\right|_{S^{\prime \prime}}=0=\left.\left(u_{2}^{\epsilon}\right)^{\prime \prime}\right|_{S^{\prime \prime}}$. Since the inverse operator $\left(-\Delta-i \partial_{t}\right)^{-1}$ exists and since the latter one is unique, cf. $[5,17]$, we have that $\left(\left(-\Delta-i \partial_{t}\right)^{\prime \prime}\right)^{-1}$ also exists and it is unique, too. Hence, we can establish the following equality

$$
\left(u_{2}\right)^{\prime \prime}-\left(u_{2}^{\epsilon}\right)^{\prime \prime}=\left(\left(-\Delta-i \partial_{t}\right)^{\prime \prime}\right)^{-1}\left(\left(-\Delta-\mathbf{k} \partial_{t}\right)^{\prime \prime}-\left(-\Delta-i \partial_{t}\right)^{\prime \prime}\right)\left(u_{2}^{\epsilon}\right)^{\prime \prime}
$$

which implies that

$$
\left\|\left(u_{2}\right)^{\prime \prime}-\left(u_{2}^{\epsilon}\right)^{\prime \prime}\right\|_{L_{p}\left(V^{\prime \prime}\right)}=\left\|\left(\left(-\Delta-i \partial_{t}\right)^{\prime \prime}\right)^{-1}\right\|_{L_{p}\left(V^{\prime \prime}\right)}\left\|\left(-\Delta-\mathbf{k} \partial_{t}\right)^{\prime \prime}-\left(-\Delta-i \partial_{t}\right)^{\prime \prime}\right\|_{L_{p}\left(V^{\prime \prime}\right)}\left\|\left(u_{2}^{\epsilon}\right)^{\prime \prime}\right\|_{L_{p}\left(V^{\prime \prime}\right)} .
$$

Since $\left\|\left(-\Delta-\mathbf{k} \partial_{t}\right)^{\prime \prime}-\left(-\Delta-i \partial_{t}\right)^{\prime \prime}\right\|_{L_{1}\left(V^{\prime \prime}\right)}$ converges to zero when $\epsilon \rightarrow 0$, we may conclude that the right hand side of the last expression also converges to zero. This fact implies that $\left(u_{2}\right)^{\prime \prime} \in L_{p}\left(V^{\prime \prime}\right)$. Moreover, we can guarantee

(i) For any two elements $\left(u_{2}^{\epsilon_{1}}\right)^{\prime \prime}$ and $\left(u_{2}^{\epsilon_{2}}\right)^{\prime \prime}$ of the fundamental family studied in Theorem 5.11 and Theorem 5.12 we can find functions $\left(g_{2}^{\epsilon_{1}}\right)^{\prime \prime},\left(g_{2}^{\epsilon_{2}}\right)^{\prime \prime} \in \stackrel{\circ}{W_{p}^{1}}\left(V^{\prime \prime}\right)$ that satisfy

$$
\left(u_{2}^{\epsilon_{1}}\right)^{\prime \prime}=\left(D_{-}^{\epsilon_{1}}\right)^{\prime \prime}\left(g_{2}^{\epsilon_{1}}\right)^{\prime \prime} \quad \text { and } \quad\left(u_{2}^{\epsilon_{2}}\right)^{\prime \prime}=\left(D_{-}^{\epsilon_{2}}\right)^{\prime \prime}\left(g_{2}^{\epsilon_{2}}\right)^{\prime \prime}
$$

and

$$
\begin{aligned}
\left\|\left(D_{-}^{\epsilon_{2}}\right)^{\prime \prime}\left(\left(g_{2}^{\epsilon_{1}}\right)^{\prime \prime}-g_{2}^{\epsilon_{2}}\right)\right\|_{L_{p}\left(V^{\prime \prime}\right)} & =\left\|\left(D_{-}^{\epsilon_{2}}\right)^{\prime \prime}\left(g_{2}^{\epsilon_{1}}\right)^{\prime \prime}-\left(D_{-}^{\epsilon_{1}}\right)^{\prime \prime}\left(g_{2}^{\epsilon_{1}}\right)^{\prime \prime}+\left(D_{-}^{\epsilon_{1}}\right)^{\prime \prime}\left(g_{2}^{\epsilon_{2}}\right)^{\prime \prime}-\left(D_{-}^{\epsilon_{2}}\right)^{\prime \prime}\left(g_{2}^{\epsilon_{2}}\right)^{\prime \prime}\right\|_{L_{p}\left(V^{\prime \prime}\right)} \\
& \leq\left\|\left(\left(D_{-}^{\epsilon_{2}}\right)^{\prime \prime}-\left(D_{-}^{\epsilon_{1}}\right)^{\prime \prime}\right)\left(g_{2}^{\epsilon_{1}}\right)^{\prime \prime}\right\|_{L_{p}\left(V^{\prime \prime}\right)}+\left\|\left(u_{2}^{\epsilon_{1}}\right)^{\prime \prime}-\left(u_{2}^{\epsilon_{2}}\right)^{\prime \prime}\right\|_{L_{p}\left(V^{\prime \prime}\right)} .
\end{aligned}
$$

As a consequence of Theorem 2.3, Theorem 5.12 and the above mentioned considerations, we may readily infer that the right hand side of the previous expression converges to zero, when $\left|\epsilon_{1}-\epsilon_{2}\right| \rightarrow 0$, i.e.,

$$
\|\left.\left(D_{-}^{\epsilon_{2}}\right)^{\prime \prime}\left(\left(g_{2}^{\epsilon_{1}}\right)^{\prime \prime}-g_{2}^{\epsilon_{2}}\right)\right|_{L_{p}\left(V^{\prime \prime}\right)} \rightarrow 0, \quad \text { when } \quad\left|\epsilon_{1}-\epsilon_{2}\right| \rightarrow 0 .
$$

Since $\left\|\left(D_{-}\right)^{\prime \prime}-\left(D_{-}^{\epsilon}\right)^{\prime \prime}\right\| \rightarrow 0$, when $\epsilon \rightarrow 0$, we conclude that $g^{\prime \prime} \rightarrow\left(g_{2}^{\epsilon_{2}}\right)^{\prime \prime}+C$, when $\left|\epsilon_{1}-\epsilon_{2}\right| \rightarrow 0$ and $\epsilon_{1}, \epsilon_{2} \rightarrow 0$, where $C \in \operatorname{Ker}\left(\left(D_{-}\right)^{\prime \prime}\right)$. Under these conditions we showed that for each $u^{\prime \prime} \in L_{p}\left(V^{\prime \prime}\right)$ there exists a function $v^{\prime \prime} \in \stackrel{\circ}{W_{p}^{1}}\left(V^{\prime \prime}\right)$ such that $u^{\prime \prime}=\left(D_{-}\right)^{\prime \prime} v^{\prime \prime}$.

(ii) Suppose that there exist two functions $\left(g_{1}\right)^{\prime \prime},\left(g_{2}\right)^{\prime \prime} \in \stackrel{\circ}{W_{p}^{1}}\left(V^{\prime \prime}\right)$ such that

$$
u^{\prime \prime}=\left(D_{-}\right)^{\prime \prime}\left(g_{1}\right)^{\prime \prime} \quad \text { and } \quad u^{\prime \prime}=\left(D_{-}\right)^{\prime \prime}\left(g_{2}\right)^{\prime \prime},
$$

are satisfied for the same function $u^{\prime \prime} \in L_{p}\left(V^{\prime \prime}\right)$. Then we have

$$
\begin{aligned}
\left(-\Delta-i \partial_{t}\right)^{\prime \prime}\left(g_{1}\right)^{\prime \prime}=\left(-\Delta-i \partial_{t}\right)^{\prime \prime}\left(g_{2}\right)^{\prime \prime} & \Leftrightarrow\left(g_{1}\right)^{\prime \prime}=\left(\left(-\Delta-i \partial_{t}\right)^{\prime \prime}\right)^{-1}\left(-\Delta-i \partial_{t}\right)^{\prime \prime}\left(g_{2}\right)^{\prime} \\
& \Leftrightarrow\left(g_{1}\right)^{\prime \prime}=\left(g_{2}\right)^{\prime \prime} .
\end{aligned}
$$

The assertion is hereby proven. 
Theorem 5.13 For each $u^{\prime \prime} \in L_{p}\left(V^{\prime \prime}\right)$, the family of $\left(P_{-, \mathcal{M}_{k, p}}^{\epsilon} u\right)$ converges to $\hat{u}^{\prime \prime}$ in $\operatorname{Ker}\left(\left(D_{-}^{\epsilon}\right)^{\prime \prime}\right) \cap L_{p}\left(V^{\prime \prime}\right)$ for all $\epsilon>0$ and $1<p<\infty$.

Proof: The proof is made in three steps: first let us consider a function $\varphi \in W_{p}^{\frac{n}{2}+1}\left(V^{\prime \prime}\right)$, a function $u \in L_{p}\left(V^{\prime \prime}\right)$, and a family of functions $\left(\left(u_{1}^{\epsilon}\right)^{\prime \prime}\right)$, where $\left(u_{1}^{\epsilon}\right)^{\prime \prime} \in \operatorname{Ker}\left(\left(D_{-}^{\epsilon}\right)^{\prime \prime}\right) \cap L_{p}\left(V^{\prime \prime}\right)$ with $\epsilon>0$ and $1<p<\infty$. Let $\epsilon_{1}$, $\epsilon_{2}>0$. In view of the decomposition (10) we have for $\left(u_{1}^{\epsilon_{1}}\right)^{\prime \prime},\left(u_{1}^{\epsilon_{2}}\right)^{\prime \prime}$ in $\operatorname{Ker}\left(\left(D_{-}^{\epsilon_{1}}\right)^{\prime \prime}\right), \operatorname{Ker}\left(\left(D_{-}^{\epsilon_{2}}\right)^{\prime \prime}\right)$, respectively.

$$
\left|\left\langle\left(u_{1}^{\epsilon_{1}}\right)^{\prime \prime}-\left(u_{1}^{\epsilon_{2}}\right)^{\prime \prime}, \varphi\right\rangle\right|=\left|\left\langle\left(u-u_{2}^{\epsilon_{1}}\right)-\left(u-\left(u_{2}^{\epsilon_{2}}\right)^{\prime \prime}\right), \varphi\right\rangle\right| \leq\left|\left\langle\left(u_{2}^{\epsilon_{2}}\right)^{\prime \prime}-\left(u_{2}^{\epsilon_{1}}\right)^{\prime \prime}, \varphi\right\rangle\right|,
$$

where $\left(u_{2}^{\epsilon_{1}}\right)^{\prime \prime}$ and $\left(u_{2}^{\epsilon_{1}}\right)^{\prime \prime}$ are elements of the fundamental family $\left(\left(u_{2}^{\epsilon}\right)^{\prime \prime}\right)$, and where $\left(u_{2}^{\epsilon}\right)^{\prime \prime} \in\left(D_{-}^{\epsilon}\right)^{\prime \prime}\left(\stackrel{\circ}{W_{p}^{1}}\left(V^{\prime \prime}\right)\right)$ for $\epsilon>0$. By Theorem 5.12 we conclude that the right hand side of the last expression converges weakly to zero, in $W_{p}^{-\frac{n}{2}-1}\left(V^{\prime \prime}\right)$, when $\left|\epsilon_{1}-\epsilon_{2}\right| \rightarrow 0$. This proves that $\left(P_{-, \mathcal{M}_{k, p}}^{\epsilon}\right)$ is a fundamental family in $W_{p}^{-\frac{n}{2}-1}\left(V^{\prime \prime}\right)$. Moreover, using the techniques and arguments presented for the family $\left(D_{-}^{\epsilon}\right)^{\prime \prime}\left(u^{\epsilon}\right)^{\prime \prime}$, with $\epsilon>0$, after Theorem 5.12 , we can refine our conclusion and conclude that the function limit belongs to $L_{p}\left(V^{\prime \prime}\right)$.

Finally, let us denote by $\left(u_{1}\right)^{\prime \prime}$ the function limit of this fundamental family. For a given $\varphi \in W_{p}^{\frac{n}{2}+1}\left(V^{\prime \prime}\right)$ with $1<p<\infty$, we have

$$
\begin{aligned}
\left|\left\langle\left(D_{-}\right)^{\prime \prime}\left(u_{1}\right)^{\prime \prime}, \varphi\right\rangle\right| & =\left|\left\langle\left(D_{-}\right)^{\prime \prime}\left(u_{1}\right)^{\prime \prime}-\left(D_{-}^{\epsilon}\right)^{\prime \prime}\left(u_{1}^{\epsilon}\right)^{\prime \prime}, \varphi\right\rangle\right| \\
& \leq\left|\left\langle\left(D_{-}\right)^{\prime \prime}\left(\left(u_{1}\right)^{\prime \prime}-\left(u_{1}^{\epsilon}\right)^{\prime \prime}\right), \varphi\right\rangle\right|+\left|\left\langle\left(\left(D_{-}\right)^{\prime \prime}-\left(D_{-}^{\epsilon}\right)^{\prime \prime}\right)\left(u_{1}^{\epsilon}\right)^{\prime \prime}(x, t), \varphi\right\rangle\right| .
\end{aligned}
$$

Theorem 2.3 and Theorem 5.13 guarantee that the first and second term of the right hand side of the last expression converges to 0 when $\epsilon \rightarrow 0$.

Summarizing, for each $u^{\prime \prime} \in L_{p}\left(V^{\prime \prime}\right)$ we have $u^{\prime \prime}=P_{-, \mathcal{M}_{k, p}}^{\epsilon} u^{\prime \prime}+Q_{-, \mathcal{M}_{k, p}}^{\epsilon} u^{\prime \prime}$. Also, we proved that

$$
Q_{-, \mathcal{M}_{k, p}}^{\epsilon} u^{\prime \prime} \rightarrow Q_{-, \mathcal{M}_{k, p}} u^{\prime \prime} \quad \text { and } \quad\left(Q_{-, \mathcal{M}_{k, p}}\right)^{2} u^{\prime \prime}=Q_{-, \mathcal{M}_{k, p}} u^{\prime \prime},
$$

which implies that $Q_{-, \mathcal{M}_{k, p}}$ is a projector and that we can define a projector $P_{-, \mathcal{M}_{k, p}}$ by

$$
P_{-, \mathcal{M}_{k, p}} u^{\prime \prime}=u^{\prime \prime}-Q_{-, \mathcal{M}_{k, p}} u^{\prime \prime}
$$

with $P_{-, \mathcal{M}_{k, p}} u^{\prime \prime} \in \operatorname{Ker}\left(\left(D_{-}\right)^{\prime \prime}\right) \cap L_{p}\left(V^{\prime \prime}\right)$. As a consequence, we have the following Hodge-type decomposition

Theorem 5.14 For $1<p<\infty$, the following decomposition

$$
L_{p}\left(V^{\prime \prime}\right)=\left(L_{p}\left(V^{\prime \prime}\right) \cap \operatorname{Ker}\left(\left(D_{-}\right)^{\prime \prime}\right)\right) \oplus\left(D_{-}\right)^{\prime \prime}\left(W_{p}^{1} \stackrel{\circ}{\left.\left(V^{\prime \prime}\right)\right)} .\right.
$$

holds. Moreover, we can define the following projectors

$$
P_{-, \mathcal{M}_{k, p}}: L_{p}\left(V^{\prime \prime}\right) \rightarrow L_{p}\left(V^{\prime \prime}\right) \cap \operatorname{Ker}\left(\left(D_{-}\right)^{\prime \prime}\right), \quad Q_{-, \mathcal{M}_{k, p}}: L_{p}\left(V^{\prime \prime}\right) \rightarrow\left(D_{-}\right)^{\prime \prime}\left(W_{p}^{1}\left(V^{\prime \prime}\right)\right),
$$

where $P_{-, \mathcal{M}_{k, p}}$ and $Q_{-, \mathcal{M}_{k, p}}$ are called Schrödinger-Bergman projectors in orbifolds.

From the preceding result we obtain the following two immediate applications

Theorem 5.15 Let $f \in W_{p}^{2}\left(V^{\prime \prime}\right)$ with $1<p<\infty$. Then the system

$$
\begin{aligned}
\left(-\Delta_{x^{\prime \prime}}-i \partial_{t}\right)^{\prime \prime} u^{\prime \prime} & =f^{\prime \prime} \quad \text { in } V^{\prime \prime} \\
u^{\prime \prime} & =0 \quad \text { at } \partial V^{\prime \prime}
\end{aligned}
$$

has a unique solution $u \in W_{p+2, l o c}^{2}\left(V^{\prime \prime}\right)$ of the form

$$
u^{\prime \prime}=T_{-, \mathcal{M}_{k, p}} Q_{-, \mathcal{M}_{k, p}} T_{-, \mathcal{M}_{k, p}} f^{\prime \prime} .
$$

Theorem 5.16 Let $f^{\prime \prime} \in W_{p}^{2}\left(V^{\prime \prime}\right)$ and $g^{\prime \prime} \in W_{p+3 / 2}^{2}\left(\partial V^{\prime \prime}\right)$ with $1<p<\infty$. Then the system

$$
\begin{aligned}
\left(-\Delta_{x^{\prime \prime}}-i \partial_{t}\right)^{\prime \prime} u^{\prime \prime} & =f^{\prime \prime} & \text { in } V^{\prime \prime} \\
u^{\prime \prime} & =g^{\prime \prime} & \text { at } \partial V^{\prime \prime}
\end{aligned}
$$

has a solution $u \in W_{p+2, l o c}^{2}\left(V^{\prime \prime}\right)$ of the form

$$
u^{\prime \prime}=F_{-, \mathcal{M}_{k, p}} g^{\prime \prime}+T_{-, \mathcal{M}_{k, p}} P_{-, \mathcal{M}_{k, p}}\left(D_{-}^{\epsilon}\right)^{\prime \prime} h^{\prime \prime}+T_{-, \mathcal{M}_{k, p}} Q_{-, \mathcal{M}_{k, p}} T_{-, \mathcal{M}_{k, p}} f^{\prime \prime} .
$$

where $h^{\prime \prime}$ is the unique $W_{p+2}^{2}\left(V^{\prime \prime}\right)$ extension of $g^{\prime \prime}$. 
Remark 5.17 All the results presented in this section can be deduced for $H_{\mathcal{M}_{k, p}, p}$.

Acknowledgement: M.M. Rodrigues and N. Vieira were supported by FEDER funds through COMPETEOperational Programme Factors of Competitiveness ("Programa Operacional Factores de Competitividade") and by Portuguese funds through the Center for Research and Development in Mathematics and Applications (University of Aveiro) and the Portuguese Foundation for Science and Technology ("FCT-Fundação para a Ciência e a Tecnologia"), within project UID/MAT/0416/2013. N. Vieira was also supported by FCT via the FCT Researcher Program 2014 (Ref: IF/00271/2014).

\section{References}

[1] R. Artino and J. Barros-Neto, Hypoeeliptic boundary-value problems, Lectures Notes in Pure and Applied Mathematics-Vol.53, Marcel Dekker, New York, 1980.

[2] S. Bernstein, S. Ebert and R.S. Kraußhar, On the diffusion equation and diffusion wavelets on flat cylinders and the n-torus, Math. Meth. Appl. Sci., 34-No.4, (2011), 428-441.

[3] M. Cahen, S. Gutt and A. Trautman, Pin structures and the modified Dirac operator, Journ. Geom. Phys., 17-No.3, (1995), 283-297.

[4] D. Calderbank, Dirac operators and Clifford analysis on manifolds with boundary, Max Planck Institute for Mathematics, Bonn, Preprint Number 96-131, 1996.

[5] P. Cerejeiras and N. Vieira, Regularization of the non-stationary Schrödinger operator, Math. Methods Appl. Sci, 32-No.4, (2009), 535-555.

[6] P. Cerejeiras and N. Vieira, Factorization of the non-stationary Schrödinger operator, Adv. Appl. Clifford Algebr., 17-No.3, (2007), 331-341.

[7] P. Cerejeiras, U. Kähler and F. Sommen, Parabolic Dirac operators and the Navier-Stokes equations over time-varying domains Math. Methods Appl. Sci, 28-No.14, (2005), 1715-1724.

[8] J. Cnops, An Introduction to Dirac Operators on Manifolds, Progress in Mathematical Physics - Vol.24, Birkhäuser, Boston, MA, 2002.

[9] R. Delanghe, F. Sommen and V. Souček, Clifford algebras and spinor-valued functions. A function theory for the Dirac operator, Mathematics and its Applications-Vol.53, Kluwer Academic Publishers, Dordrecht etc., 1992 .

[10] C. Farsi, Dirac operators on non-compact orbifolds, J. Geom. Phys., 59-No.2, (2009), 197-206.

[11] T. Friedrich, Zur Abhangigheit des Dirac-operators von der Spin-Struktur, Colloq. Math., 48, (1984), $57-62$.

[12] K. Gürlebeck, K. Habetha and W. Sprößig, Holomorphic functions in the plane and $n$-dimensional space, Birkhäuser Verlag, Basel, 2008.

[13] K. Gürlebeck and W. Sprößig, Quaternionic and Clifford calculus for physicists and engineers, Mathematical Methods in Practice, Wiley, Chichester, 1997.

[14] R. S. Kraußhar, Generalized analytic automorphic forms in hypercomplex spaces, Frontiers in Mathematics, Birkhäuser, Basel, 2004.

[15] R. Kraußhar, Dirac and Laplace operators on some non-orientable conformally flat orbifolds, J. Math. Anal. Appl., in press.

[16] R.S. Kraußhar and J. Ryan, Some conformally flat spin manifolds, Dirac operators and Automorphic forms, J. Math. Anal. Appl., 325-No.1, (2007), 359-376. 
[17] R.S. Kraußhar and N. Vieira, The Schrödinger equation on cylinders and the n-torus, J. Evol. Equ., 11-No.1, (2011), 215-237.

[18] N.H. Kuiper, On conformally flat spaces in the large, Ann. Math., (2)-50, (1949), 916-924.

[19] H.B. jun. Lawson and M.L. Michelsohn, Spin geometry, Princeton Mathematical Series-Vol.38, Princeton University Press, Princeton, NJ, 1989.

[20] P. Petersen, Riemannian Geometry, Graduate Texts in Mathematics - Vol.171, Springer Verlag, New York, 1997.

[21] J. Ryan, Conformal Clifford manifolds arising in Clifford analysis, Proc. R. Ir. Acad., Sect. A 85, (1985), $1-23$.

[22] T. Tao, Nonlinear dispersive equations, local and global analysis, CBMS Regional Conference Series in Mathematics-Vol.106, American Mathematical Society, Providence, RI, 2006.

[23] N. Vieira, Theory of the parabolic Dirac operator and its applications to non-linear differential equations, PhD Thesis, Univerity of Aveiro, 2009. 